\title{
Conscious Thought Is for Facilitating Social and Cultural Interactions: How Mental Simulations Serve the Animal-Culture Interface
}

\author{
Roy F. Baumeister and E. J. Masicampo \\ Florida State University
}

\begin{abstract}
Five empirically based critiques have undermined the standard assumption that conscious thought is primarily for input (obtaining information from the natural environment) or output (the direct control of action). Instead, we propose that conscious thought is for internal processing, to facilitate downstream interaction with the social and cultural environment. Human consciousness enables the construction of meaningful, sequential thought, as in sentences and narratives, logical reasoning, counting and quantification, causal understanding, narratives, and the simulation of events (including nonpresent ones). Conscious thought sequences resemble short films that the brain makes for itself, thereby enabling different parts of brain and mind to share information. The production of conscious thoughts is closely linked to the production of speech because the human mind evolved to facilitate social communication and information sharing, as culture became humankind's biological strategy. The influence of conscious thought on behavior can be vitally helpful but is mostly indirect. Conscious simulation processes are useful for understanding the perspectives of social interaction partners, for exploring options in complex decisions, for replaying past events (both literally and counterfactually) so as to learn, and for facilitating participation in culture in other ways.
\end{abstract}

Keywords: consciousness, culture, cognitive evolution, mental stimulation

Understanding consciousness has been one of the grand perennial problems with which multiple disciplines have contended. The longstanding, simple, naïve view has held that the conscious self is fully in charge of behavior, sees the world generally as it is, and directs behavior as it sees fit. This view has come under criticism from a long line of thinkers, stretching back at least to Nietzsche and Freud, up to current researchers such as Bargh, Dijksterhuis, and Wegner. The critiques and contrary evidence have been so thoroughly devastating that some have begun to wonder openly whether conscious thought has any usefulness at all (e.g., Bargh, 1997; Pocket, 2004).

This article undertakes a positive approach to the purpose and function of conscious thought. We are prepared to concede the correctness of much (not all) of the negative evidence against it, but we think that is generally beside the point. If conscious thought is indeed useless, irrelevant, and even counterproductive for some tasks, then perhaps its adaptive value is to be found elsewhere. We look at it as an adaptation suited to the relatively sophisticated demands of the unique kinds of social life that humans develop, including culture. Many theories have assumed that conscious

Roy F. Baumeister and E. J. Masicampo, Department of Psychology, Florida State University.

We thank Kathleen Vohs, Ezequiel Morsella, Jonathan Schooler, David Hamilton, Martin Seligman, Larry Barsalou, Mike Kaschak, Sarah Ainsworth, Eric Garland, and the philosophy and psychology faculty at Bates College for very helpful comments. We thank the Templeton Foundation and the National Institutes of Health for grant support.

Correspondence concerning this article should be addressed to Roy F. Baumeister, Department of Psychology, Florida State University, Tallahassee, FL 32306-4301. E-mail: baumeister@psy.fsu.edu thought is for perceiving the environment and for directly controlling action, but the detractors have revealed its inadequacies for those functions. Instead, we suggest that it serves the vital interface between the animal body and the cultural system and that its powers are best appreciated in terms of simulating events away from the here and now. These simulations could include replaying past events (even counterfactually) to learn from them, imagining possible courses of future action and their potential consequences, and empathically intuiting the perspectives and mental states of interaction partners.

Theorists across a number of disciplines distinguish between two forms of consciousness. The first, phenomenal awareness, describes feelings, sensations, and orienting to the present moment. It is essentially the way living things with brains obtain information from the environment. The general view is that this lower level of consciousness is much older in phylogeny and is present in many if not all animals (Mendl \& Paul, 2004; Panksepp, 2005). The second form of consciousness involves the ability to reason, reflect on one's experiences, and have a sense of self, especially one that extends beyond the current moment. Researchers have argued that this type of consciousness is unique to humans (Damasio, 1999; Edelman, 2004) and is perhaps characterized most centrally by an ability to simulate events beyond the here and now, such as through mental time travel (Suddendorf, 2006).

The current article is concerned primarily with the second form of consciousness that is largely specific to humans. We refer to it as conscious thought. To the extent that the capacity for conscious thought is uniquely human, it may well be linked to and perhaps useful for the sorts of behaviors that are also uniquely human Phenomenal awareness is also important; we assume that integrating sensory input and action control makes use of that more basic 
form of consciousness. Moreover, conscious thought must work with that system to adapt the old, animalistic ways of doing to the new demands of the cultural environment (e.g., Damasio, 1999; Edelman, 2004; Panksepp, 2005). Still, we focus mainly on the contributions of conscious thought.

Several major lines of thought have paved the way for the present analysis. Mead (1934, p. 18) asserted that social life is a precondition for consciousness, and Vygotsky (1962) proposed that learning to think is heavily influenced by learning to talk. Humphrey $(1976,1986,2006)$ proposed that conscious thought mainly serves the purpose of enabling people to understand each other. To be sure, there are differences. Humphrey's approach, for example, falls in the so-called "theory theory" of mind reading, by which people use introspection to formulate a theory about how minds work and then apply that to others, whereas our approach is more consistent with its rival, simulation theory (see Gallese \& Goldman, 1998).

As a point of departure, we next discuss some of the criticisms of conscious thought that our theory must accommodate. The opposing views that currently dominate the debate about conscious thought can be caricatured as asserting that the conscious self does everything versus that it does nothing. A debate between the omnipotent and impotent depictions of conscious thought will result in an impasse. Almost certainly, we think, an intermediate position will be correct. If so, then the challenge is to identify what conscious thinking can do, given a sober understanding of what it cannot do or cannot do very well. Hence, we begin with its limitations.

\section{Five Criticisms of Conscious Thought}

The naïve view that the conscious self knows all about what the person is doing and can direct behavior at will has come under fire from several well-thought-out and empirically supported perspectives. Here, we summarize five major criticisms of conscious efficacy. Each calls into question the view that the conscious self does everything, and so, if the only alternative is that the conscious self does nothing, then they support the latter (do-nothing) position. In our view, however, they point the way toward a theoretical understanding of the properly delimited function of conscious thought.

These challenges must be dealt with if one is to resurrect any efficacy for conscious thought. Any new theory that posits value or efficacy to conscious thought must incorporate or refute these points.

First, conscious explanations of one's own behavior are sometimes wrong, as explained in a classic article by Nisbett and Wilson (1977). People frequently introspect and then tell someone why they did what they did, but their reasons can be demonstrably false. In fact, people's explanations often seem drawn simply from a common trove of stock explanations, ones that are collectively deemed plausible by the social group, rather than from true introspection. The implication is that consciousness does not know what goes on inside its own psyche to produce behavior and that it will invent false explanations (borrowed from the collective stockpile of group beliefs) to cover its ignorance, then passing them off as if true. What is worse, it often ends up believing its own false explanations.
Second, conscious thought may be too slow to guide behavior online. Libet (1985) showed that when people make an arbitrary decision to initiate a motor response, brain activity has already been on the rise for part of a second before the conscious decision. So, brain processes commence action first, then conscious thought joins in.

The implications of Libet's findings have been debated fiercely. They are often interpreted to mean that conscious thought cannot cause behavior because the response is already underway before the initiating decision shows up in consciousness. Libet (2004) himself rejected that explanation and suggested that although initiation may take place outside of behavior, the conscious mind does enter in time to veto the process. Still, the conscious decision to initiate a response seems not to be the true start of the process. This strikes a serious blow against the view that consciousness is responsible for initiating and controlling behavior.

Third, conscious thought produces false explanations to integrate information about the world. Gazzaniga's (e.g., Gazzaniga, 2000, 2003) split-brain patients would invent integrative explanations that were sometimes obviously and even laughably false. $\mathrm{He}$ concluded that conscious thought is not really understanding the world but just inventing convenient and possibly false explanations. Insofar as a false understanding is normally a poor basis for action, the pattern further confirms the uselessness of conscious thought.

Fourth, conscious thought is dispensable in the initiation of action. Bargh's (e.g., Bargh, 1997, 2006; Bargh \& Chartrand, 1999) program of research has focused on showing that many behaviors once thought to be the exclusive province of conscious guidance can be produced by nonconscious, automatic processes, in some cases without any conscious realization of what is happening. ${ }^{1}$ The pursuit of a wide range of goals and social motives, for instance, can be activated in the absence of a conscious decision to do so. After being exposed to nonconscious reminders of a goal, people tend to pursue the goal with as much vigor and ultimate success as those who are told explicitly to pursue the same objective (Bargh, Gollwitzer, Lee-Chai, Barndollar, \& Trötschel, 2001). Many who read or hear about this research come away thinking that consciousness is useless and unnecessary, although this may be a caricature of the work. Regardless, the fact that conscious processing is not needed for many important categories of behavior has clearly and substantially narrowed the scope of things for which conscious thought is truly needed, and it is fair to wonder whether there are any such behaviors that cannot be produced without the active guidance of the conscious mind.

Last, conscious thought can be mistaken about whether oneself caused some effect on the environment (Wegner, 2002). Conscious thought seems to infer, based on cues, that the self has acted, rather than having direct and accurate knowledge of what actions are being initiated and controlled. The conscious mind infers that the self has acted by noticing that some thought preceded action by an

\footnotetext{
${ }^{1}$ To be sure, the absence of conscious processing can be overstated. Success at these experiments is normally predicated on considerable conscious thought by the researchers, who design the procedures carefully. Moreover, instructions are communicated to the participants via conscious thought. Still, participants remain consciously unaware of some crucial aspects of the causation of their own behavior.
} 
appropriately brief moment. The person will sometimes take responsibility for doing things that were in fact not done by the person. The implication is that consciousness is not directly involved in controlling those actions, which is again a sign that it is more of a spectator (and an easily fooled one at that) than a participant.

Some defenders of consciousness have noted logical flaws and overstatements in these critiques (e.g., Mele, 2009). For example, the instruction to initiate a meaningless, unplanned, and arbitrary finger movement in Libet's procedures may have ruled out many important opportunities to do anything useful. Likewise, it is a long leap from noting occasional errors in conscious thought to concluding that its contents are generally wrong or from finding that particular acts can be performed without conscious intention to concluding that most or all acts are performed independently of conscious intention. Still, for the sake of argument, we are ready to concede that these critiques all have substantial merit and are at least partly correct.

Taken together, these critiques indicate that the conscious mind sometimes holds and believes false ideas. (The many standard perceptual illusions point to the same conclusion.) The pragmatic utility of false explanations is dubious at best. Insofar as consciousness invents false explanations about self and world, it cannot be much help in dealing with them. One could argue back that sometimes its explanations are correct and therefore can sometimes be helpful. Against that view, conscious explanations lack reliability and credibility if they are sometimes wrong, especially given that the conscious mind does not know when it has come up with a false explanation. Conscious thought cannot afford to trust itself.

Apart from the occasional wrongness and resulting unreliability, these critiques convey a further problem. If the automatic mind has much or all of the necessary information, what added value is gained from conscious processing? The eye sees the bear or the dollar or the hamburger and conveys this to the brain, which initiates appropriate action. All of this could take place nonconsciously. It is hard to see what benefit there would be from adding conscious thought to the process.

The five critiques can be sorted into two groups. The first, third, and fifth critiques note that conscious understandings and explanations are often wrong and thus that conscious thought does not seem to contribute positive value to the perception of self and world. The second, fourth, and fifth critiques question its contribution to the control of action. Together, these are potentially devastating to the general assumption that the value of conscious thought lies in perceiving self and world and in controlling action. A new theory must look beyond these old assumptions for a viable function of conscious thought.

\section{Looking Elsewhere}

To provide a theory of the positive value of conscious thought that can avoid the problems raised by the five critiques, we propose that conscious thought is not primarily for the sake of direct action control and/or sensory input. Indeed, it may not be all that necessary for dealing directly with the physical environment at all. As a very rough and approximate generalization, we suggest that phenomenal awareness enables a simple animal to coordinate its actions with the physical environment-whereas conscious thought enables human beings to coordinate with their social and cultural environment.

One may easily get the impression that the purpose of conscious thought is for guiding one's present actions because it often focuses on what is happening here and now. However, we think its more distinctive function is to simulate events away from the here and now. People focus an average of $30 \%-40 \%$ of their thoughts on concerns that are unrelated to their present behaviors (Klinger \& Cox, 1987), and some people's minds wander from the here and now more than $90 \%$ of the time (Kane et al., 2007). Even when conscious thoughts are tied to current behaviors, they are often used for the purpose of recalling similar behaviors from the past, anticipating the consequences of present behaviors, or pondering alternative courses of action. The eyes and ears can perceive the present environment, and they may do so without needing help from conscious thought, but the eyes and ears are not much help if the animal wants to replay the past, anticipate the future, or imagine hypothetical scenarios.

The content of conscious thought is presumably the result of prior causes. It may have emergent properties, especially insofar as there are styles of thought that depend on consciousness. Conscious thoughts may therefore have important causal effects on subsequent behavior (for a review, see Baumeister, Masicampo, \& Vohs, in press). Nonetheless, we see conscious thought as largely created and determined by automatic processes. The brain constructs a short movie for itself, as it were. ${ }^{2}$ Impulses to act begin in the nonconscious parts of mind and brain, and they may or may not pass through consciousness. Moreover, whether they pass through conscious thought is also likely determined by a nonconscious supervisory process.

Our approach is guided by the assumption that most of the distinctively human traits are adaptations for the distinctively human forms of social life, including culture (Baumeister, 2005; Mead, 1934). Culture coevolved with the human psyche, thereby being part of the selection environment that would favor individuals with higher genetic competence for cultural participation (Boyd \& Richerson, 1985, 1995; Jablonska \& Lamb, 2005). Several theories have argued that primate intelligence evolved to meet escalating demands and opportunities for social competence (Byrne \& Whiten, 1988; Dunbar, 1998), and human culture continued this line (Tomasello, 1999). Culture is based on sharing information, coordination of interactive roles in flexibly organized social systems, and mutually beneficial economic exchange (Baumeister, 2005). Hence, traits such as theory of mind and empathy, complex learning, future-oriented decision processes, self-regulation and rule following, and flexible selfhood would be useful for culture. We think that the value of human conscious thought is more likely to be found in such activities than, Libetstyle, in direct initiation of muscle movements.

In this article, we develop the view that the special capabilities of human conscious thought serve crucial functions for enabling the advanced forms of social life humans have, including culture. We discuss consciousness as a place where the mind constructs meaningful sequences of thought, especially simulating events

\footnotetext{
${ }^{2}$ For fans of Dennett's (1991) approach, we note that these films can well occur in multiple places in the brain rather than entailing a single Cartesian theater.
} 
away from the here and now, and then we describe how that capability serves those social and cultural functions.

\section{Conscious Thought Plays a Supporting Role}

The current theory emphasizes that conscious thought is a tool of older processes, such as nonconscious and automatic ones. Thus, we reject both extreme views, that conscious thought represents the driver in full charge of behavior and that it is a helpless passenger merely along for the ride. Instead, conscious thought is like a fancy new navigational system. Many dual process theories of mind distinguish between one process that is conscious and controlled and another that is nonconscious and automatic, with behavior being caused by either one process or the other. Rather than consider the two systems as discrete and as causing behavior at different times, we suggest that conscious thought represents a relatively new system that works within the framework of the older system (see Figure 1). Thus, we propose that in the final analysis, the proximal causes of all behaviors are nonconscious (Rosenbaum, 2002) - but sometimes their execution is informed by prior conscious thought.

From this alternative perspective, evidence that a stimulus or event can cause a behavior unconsciously says nothing about whether conscious thought can sometimes play a causal role. Conscious thought may serve as an adaptive tool for older, unconscious processes by providing input that influences the central executive. Some would say this constitutes free will, though, no doubt, others would object to the term. For present purposes, the important point is that the operations of conscious thought can have an indirect but genuine impact on behavior.

Conscious thought is thus neither all-powerful nor utterly powerless. As social animals evolved into cultural ones, new mental structures may have been needed to enable the animal to deal with the culture. Yet these do not necessarily entail that the nonconscious executive turned over control and authority to the new structures. Instead, conscious thought is likely to provide helpful input to the same executive. Hence, one should ask what sort of help conscious thought could provide, rather than assuming that it took over the reins of power and supplanted the age-old automatic system of control.

Thinking of conscious thought as a tool of much older processes rather than a direct executive helps resolve several of the problems we noted above. Libet's (1985) ostensible findings that impulses to act do not originate in conscious thought are beside the point. We agree that the impulse originates in the automatic system. The role of conscious thought is to reshape (Baumeister, Vohs, DeWall, \& Zhang, 2007) and reprogram (Gollwitzer, 1999) those automatic responses through input from culture, as well as to simulate the event mentally before doing it-perhaps also discussing it with real or imagined other people. All this is done to ascertain whether acting out the impulse would be a good idea (to use the common and revealing phrase!).

Likewise, the many findings that behavior can be generated without conscious thought (see Bargh, 2007, for a review) dovetail well with the view of conscious thought as a supplementary process. The impact of conscious thought as simulation is almost by definition indirect, and so, the direct, proximal causes of behavior will always be automatic and nonconscious. For example, the proximal cause of any muscle movement is likely to consist of nonconscious physical events such as neuronal firings. Hence, researchers in the Bargh tradition can produce complex behaviors by bypassing conscious awareness. The influence of conscious thought can be bypassed precisely because it is upstream in the causation of behavior.

Conscious thought is for incorporating knowledge and rules for behavior from culture. Over time, automatic responses then come to be based on that new input. The impact of conscious thought is thus likely to be seen only at the macro level, in temporally large units. Galdi, Arcuri, and Gawronski (2008) demonstrated this point nicely in a recent study. They asked participants to take a stance on a controversial issue after reading talking points from both sides of the debate. Then they assessed participants' beliefs and associations 1 week later. Participants' automatic associations did not significantly match up with their consciously reported beliefs on the day that they read about and took a stance on the debate. However, consciously reported beliefs predicted automatic associations 1 week later. Apparently, the consciously endorsed belief caused one's automatic responses to change slowly over time.

Last, the treatment of conscious thoughts as playing an upstream, advisory role incorporates even the contributions by Wegner (2002). He showed that people sometimes mistakenly believe that their thoughts are responsible for actions that were in fact caused by external factors, such as the preprogramming of the laboratory computer on which responses were registered. One might plausibly (though wrongly) infer that these thoughts caused the executive to act when one sees the body doing precisely what one's thoughts suggested-even if, in reality, the unconscious executive processes disregarded the conscious thoughts and took that same course of action for completely unrelated reasons. The indirectness of conscious influence makes such errors possible.

To illustrate, suppose an advisor recommends that the king sponsor a parade, and the king ignores the advice but sponsors the parade anyway to impress his mistress. The advisor, like Wegner's conscious self, might mistakenly believe that his thinking and advice caused the parade to happen. The mistake proves the
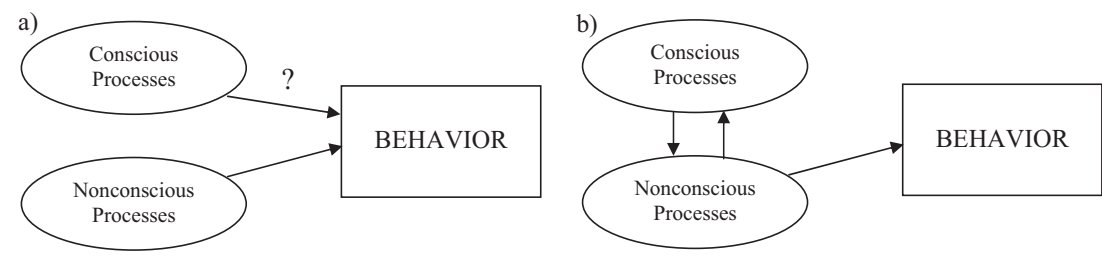

Figure 1. Conscious processes are a tool of older, nonconscious ones (Panel b), and so, conscious processes are not the direct cause or controller of behavior (Panel a). 
indirectness of the advisor's control, which is what Wegner's work established. Still, this sort of mistake would not likely be costly or problematic for either the king or the advisor.

\section{Brain Making Input Into Itself}

Conscious thought, in this view, is not the brain's executive but rather a means by which the brain can gain useful new insight from the pool of information it already has. Conscious experience is the brain making input into itself. Admittedly, that sounds paradoxical. Why would a brain make input into itself? After all, as Dennett (1991) and others have pointed out, it already has all the information it uses to make conscious thought, so what is to be gained by using that information to make an internal movie as opposed to proceeding directly with action and other business? More broadly, what value or power do thoughts gain by being conscious?

There are three parts to the answer. They can be labeled mental crosstalk, social communication, and sequential simulation. Mental crosstalk is the notion that conscious thoughts enable information from distributed, parallel processing in different brain and mind sites to be shared and integrated. Social communication refers to being able to tell one's thoughts to other people (and learn theirs). Sequential simulation is the idea that conscious processing makes possible some kinds of thinking (e.g., logical reasoning) that are different from and in some ways better than what unconscious minds can do. The next sections of this article elucidate these three answers.

\section{Crosstalk: Toward the Conscious Mind}

Why are there brains? Above all, brains facilitate survival and reproduction by making helpful links between sensory input and motor output. If adaptive behavior consists of making the optimal behavioral responses to changing circumstances, the brain is crucial: The brain is where stimulus meets response.

Therefore, the basic design problem for a brain is to link each stimulus to the relevant response-that is, to get the important incoming information about the environment connected to the proper place(s) in the brain where relevant knowledge is stored and optimal responses are prescribed. This could be a simple matter of sorting, as long as there are only a few types of stimuli and responses. However, as the brain becomes larger and more complex, with more different sets of information stored in various places, the difficulty of this problem (of linking stimulus to optimal response) increases exponentially. Connecting the incoming stimulus with all (or even most) of the possibly relevant brain sites could be difficult.

The apparent solution was to broadcast an information-rich signal out to much of the brain. Thus, the brain takes incoming information, fashions a message, and displays it internally so that associations can activate relevant stores of information. This is the beginning of consciousness, according to Baars's (1997, 2002, 2003) global workspace theory. As his account emphasizes, consciousness has limited capacity but broad access. The limited capacity refers to the amount of information that can be broadcast at any one time. The broad access refers to the fact that what is displayed in conscious thought is able to reach and activate a large amount of information stored in separate places in the mind. In ordinary experience, people think about some topic by holding the central thought (e.g., an upcoming trip) in the conscious mind, and other associated thoughts (e.g., what needs to be packed) pop into awareness.

Moreover, once information is retrieved from scattered sites, there is no guarantee that these will all favor the same course of action. Hence, some workspace is needed where conflicting prescriptions for behavior can be resolved. Morsella (2005) noted that many inner conflicts are resolved without consciousness even in humans. For example, the two eye pupils remain dilated to the same degree even if one eye looks at bright light and the other is shut. Morsella concluded that conflicts become conscious when they pertain to muscles that will move bones, so the animal can act in a unified manner.

The view of consciousness as resolving inner conflicts so that skeletal muscles can agree on how to move the body pertains to both phenomenal awareness and conscious thought. In simple animals, motivational conflicts and other behavioral decisions may be paramount. Thus, when being chased, the animal's impulse is to move in any direction away from the predator, but it is still necessary to choose rapidly among different possible directions and navigate around obstacles. We emphasize also that awareness is not necessarily extended deeply into inner processes but rather serves the function of coordinating the inner processes with the environment. The animal's awareness does not need to extend to each tiny muscle in the left hind leg-rather, it helps coordinate the general running with the need to jump over a specific rock.

Thus, the first benefit of conscious processing is based on the dispersion of information in memory and, hence, the distribution of processes across the brain. Both types of consciousness, namely, phenomenal awareness and conscious thought, enable the different parts of the mind and brain to share information with each other (Baars, 1988; Crick, 1984; Dehaene, Sergent, \& Changeux, 2003; Edelman, 2004). Consciousness has been seen by almost all theorists as helping to integrate information (see Morsella's, 2005, discussion of the integration consensus).

Social life increases the amount and diversity of information that needs to be integrated (and its conflicts resolved) to permit action, so the more social an animal is, the more powerful its conscious capabilities need to be. Even the mere number of brain sites relevant to a particular action is vastly increased by social life. Research by Dunbar (1998) and others has suggested that the expansion of brain size, even in nonhuman animals, was driven by the demands of coping with the social environment. Social life created many new layers of potential complexity needing sophisticated storage in the brain memory, as groups developed hierarchies and increasingly differentiated roles, factions, and rivalries, and as knowledge about the behavioral tendencies of others could be helpful in social strategizing (Byrne \& Whiten, 1988; Humphrey, 1976).

Also, crucially, social life and especially culture produce new classes of inner conflict that may require an integrating consciousness to resolve. Culture has rules and other prescriptions that require an animal to overcome its immediate and self-interested impulses. To act in a civilized manner is precisely not to act like an animal, in the colloquial phrase. Conscious thought, as opposed to mere phenomenal awareness, may be needed to handle this advanced class of conflicts.

For example, consider all of what is involved in responding to an offer of cheesecake for dessert at a dinner party: politeness, 
hunger, craving, knowledge about calories, declining tolerance for lactose, the need to avoid spilling food on one's expensive clothes, the possibility of offending the cook, the impressions others may draw based on one's response, and so forth. Insofar as these concerns are scattered across different brain sites, something is needed to activate all of them, integrate their responses, and resolve their conflicting recommendations so that a single response can be made. Consciousness may provide the crucial mechanism by which the helpful wisdom of different sites can be integrated without their all being linked together in advance.

The adaptive utility of this inner process depends on reaching all the relevant sites. Essentially, consciousness holds up some incoming information and asks the scattered brain sites, "Do any of you have ideas for what to do about this?" As natural selection increased the size of the brain, it is in principle possible that the inner signal would remain about the same, but more plausibly, there would be two related types of changes. First, the signal might become stronger and more vivid (corresponding to asking that question more loudly). As with any broadcast, the farther it has to reach, the more powerful the signal must be.

Second, and more important, the quantity of information contained in the signal would likely increase. This corresponds to asking the question in a more detailed manner. The entire mental system would be more efficient to the extent that the signal broadcast across the brain has plenty of useful information. The likelihood of activating any positive response and especially of activating all the needed positive responses is high to the extent that the "this" (in the question of what to do about this) is already clearly defined and understood. This has important implications. Cleeremans (2006) summarized multiple lines of evidence and theory equating consciousness with high strength and quality of cognitions. The view that conscious thoughts differ from unconscious ones partly by virtue of being stronger and higher in quality (specifically, clear, distinctive, and stable) seems to be well established. We shall return to the notion of conscious thoughts as having high quality as the third benefit of consciousness.

\section{Social Communication, Culture, and Conscious Thought}

The previous section proposed inner mental crosstalk as the first benefit of conscious thought. The second benefit is that people can talk about their thoughts with each other. By definition, people cannot report on their unconscious thoughts. For two people to discuss a plan, they must have a shared mental representation of the plan and must be able to access their thoughts so as to tell them. Such discussion will enable them to comment on each other's ideas, correct each other's mistakes, and reach an agreement that will enable them to carry the plan out together.

Conscious thought is often synonymous with reportable states. Many researchers implicitly or explicitly state that the best operational definition of consciousness is that it consists of mental states and events that the person can report to others (e.g., Baars, 1997, p. 11; Nisbett \& Wilson, 1977). This view has sometimes been questioned with reference to phenomenal awareness (Block, 1995, 2007). Conscious thought, however, is often defined unequivocally in these terms (Bayne \& Chalmers, 2003; Izard, 2009). Generally (and oddly), though, theorists seem to overlook the plausible implication - that a central function of conscious thought is precisely to enable people to report their mental states and events to others. We assume that nearly all talking is conscious. Being able to tell one's thoughts to others has at least two vital advantages. First, it facilitates group action (e.g., H. Clark, 1996). Second, it enables an individual's thoughts and actions to benefit from and be modified according to the wisdom of others.

In fact, several prominent thinkers (e.g., Vygotsky, 1962) have proposed that much of conscious thought originates as silent speech. William James's dictum that thinking is doing (James, 1890, Vol. 2, p. 333) has reigned as a truism in psychology for more than a century. In a trivial sense, it is indisputably true. However, we propose that a more precise and accurate formulation would be that much of conscious thinking, at least, is for talking. Instead of searching for the functions of conscious processes in how they enable the person to act, we propose a more thoroughly social basis, namely, in changes in interaction based on communication and mutual understanding.

\section{From Primate to Human Conscious Thought}

Nonhuman primates possess many cognitive abilities that humans do. The major differences lie in the human improvements in identifying with each other and understanding each other, which, when added to the primate mental abilities, "transformed them into new, culturally based cognitive skills with a social-collective dimension" (Tomasello, 1999, p. 7). Herrmann, Call, HernandezLloreda, Hare, and Tomasello (2007) administered a battery of mental tests to human children, chimps, and orangutans, and they confirmed that the three groups were largely similar on most tests-but differed consistently and significantly on socialcognitive skills. More generally, primates (especially chimps) can pass some tests of theory of mind but not others (see also Call \& Tomasello, 2008; Corballis, 2004; Hauser, Chomsky, \& Fitch, 2002).

Hence, group action in primates is limited and does not show the kind of intentional collaboration that is common to almost all human groups. As summarized by Tomasello, Carpenter, Call, Behne, and Moll (2005), the crucial advances that enabled human minds to create culture consisted of acquiring the ability to understand the inner, mental states of others and the motivation to communicate one's own inner thoughts to others. These changes, we think, transformed phenomenal awareness into human conscious thought.

The sort of changes that might have facilitated such advances would include the so-called mirror neurons in the brain. Monkeys and other primates have such neurons, which respond to the actions of other animals as if the perceiver were acting (Gallese \& Goldman, 1998). Mirror neurons are especially sensitive to arm movements, and crucially, they react to the goal of the act rather than just the muscle movement. As Gallese and Goldman (1998) argued, mirror neurons thus provide the basis for an incipient theory of mind, insofar as they register another animal's intention (e.g., one monkey sees that the other was reaching for that banana, even though it came away empty-handed). Such mirror perception also rests on going beyond direct observation via mental simulation and pattern completion, which we argue create some of the particular powers of conscious thought.

Rudimentary theory of mind based on mirror neurons responding to arm movements may have led to communication by gesture. 
The case is reasonably strong that gestural communication preceded vocal speech (see especially Corballis, 2009). As one sign, some nonhuman primates today can learn sign language, but none can achieve vocal speech. Even human pairs who lack a common language still fall back on gestures to communicate, with some success. Early hominid communication may have used increasingly specific gestures (sign language) augmented by vocal sounds, which over time was reversed so that, as today, people communicate mainly by speech while still using hand gestures to enhance expression. The evolution of the human brain for speech may have caused the prevalent right-handedness that is normal among humans but not found in other species (Corballis, 2003; see also Gazzaniga, 2000, on greater lateralization in humans than in other species).

Early evolutionary theorists assumed that the big brain was the basis for human evolution and led to such things as upright posture, but the fossil record discredited this by showing that the upright posture preceded the large brain (e.g., Gould, 1977). Australopithecus stood upright but had a relatively small brain. This raised two questions: Why the upright posture? What did finally get the brain expanding?

The not entirely satisfactory answers regarding upright posture included freeing the hands for carrying things and making fire. (Extensive tool use came later.) The use of hands for gestural communication may well be a major part of the answer, however. Hominids who used their hands to communicate would then be able to use and share increasingly large quantities of information, which is what humans did. The proliferation of information would likely have created selection advantages for the larger brainshence the subsequent expansion in brain size.

A crucial phase in human evolution came with the cooling of world climate (especially Africa) during the early Pleistocene era, which moved many human groups from forests to grasslands. There, they encountered big cats and other predators who not only posed a danger to the humans but also competed with humans for prey (see Corballis, 2009). Instead of evolving catlike physical traits to compete as equals, humans appear to have evolved to use social and cognitive traits to compete (see also Tooby \& DeVore, 1987). Communication would have been central to any such strategy because it is both social and cognitive and enables the cognition to have a social dimension (Tomasello, 1999).

The later shift from gesture to speech as a medium of communication was a decisive step in human evolution. Corballis (2004) argued that speech rather than gestural language enabled culture to progress, given the multiple advantages of speech. These advantages included using less physical energy, being effective at night, not presupposing that the intended audience is already looking, and perhaps most important, freeing the hands for doing other things, so that groups of humans could talk while using their hands for other tasks, including hunting, using tools, and holding babies.

Vocal speech could thus facilitate group action (H. Clark, 1996), and most likely, it effected sweeping transformations of it. By way of contrast, collective action in many groups of animals must occur without prior discussion. One starts, and others join in. Human groups have demonstrably more complex processes. These include group conversation, voting, and rational allocation and performance of complementary roles, not to mention committee meetings and invocation of agreed-upon rules and regulations. All of these depend on communication.
Thus, a major evolved function of the human conscious mind is to understand the group's communications. Such understanding enables individuals to access the group's collective store of information (the latter being one of the essential advantages of culture) and, on that basis, to participate in its organized activities. Conscious thought enables people to work together in new and better ways, based on communication.

We noted earlier that Tomasello et al. (2005) proposed two crucial changes from ape mind to human: the understanding of others' inner states and the drive to express one's own states. The latter may be reflected in inner speech, by which the mind constructs a running verbal commentary on the body's activities. Gazzaniga $(2000,2008)$ coined the term the left-brain interpreter to describe the operation of certain brain areas for producing an inner running narrative of one's life. He suggested its function is for furnishing an autobiographical coherence and sense of self, but we suggest a more important function is to render one's activities and experiences in language so that they can be readily shared with others. Hence, we suspect that the left-brain interpreter emerged to serve a social function rather than an intrapsychic one.

\section{Talking Transforms Thinking}

Conscious thought, as experienced today, is typically filled with silent speech (alongside some other content). To understand what mental processes produce conscious thought, we think it is most relevant to understand the processes that produce speech. Keeping thoughts in one's mind would be an additional step of inhibiting the vocal output, such as when predators or prey are near. Vygotsky (1962) was an early exponent of the view that learning to talk is a large influence on learning to think (at least thinking in words; nonverbal thinking in images precedes both). He illuminated the relationship between thought and speech by studying how each develops in children. A child acquires speech by learning single words first. Later, the child learns how to connect them and eventually to use whole sentences. Speech develops from simple, individual units to coherent and colorful wholes.

The development of thought is greatly advanced and shaped by the acquisition of speech, in Vygotsky's view. A child's first thoughts are whole, complex images. It is only over time and with the help of language that the child is able to break each complex thought into its various components: I want him to share with me, but he is being greedy.

Much of thought, then, derives from talking (though thinking is not entirely absent without language). Indeed, it is well established that children learn first to read aloud and only later to keep quiet (Crowder \& Wagner, 1991). Electromyographic evidence indicates that silent processing of language continues to activate speech muscles, quite strongly in 6-year-olds and then less with age (McGuigan \& Bailey, 1969; McGuigan, Keller, \& Stanton, 1964). Even in adults, silent thought continues to evoke implicit movements of speech muscles (McGuigan, 1970). Further evidence of the functional resemblance of thinking and talking comes from a recent meta-analysis by Fox, Ericsson, and Best (2009). Assigning people to perform an extra task while solving problems would normally reduce success at the problems, but this metaanalysis found across dozens of studies that assigning research participants to verbalize their thoughts while solving problems had essentially no effect on performance, as compared to performing 
the same tasks silently. In a similar vein, recent studies have shown that suppressing inner speech interferes with some executive function activities such as task switching (Emerson \& Mikaye, 2003; Miyake, Emerson, Padilla, \& Ahn, 2004).

\section{Culture, Communication, and Conscious Thought}

Our analysis thus elaborates Tomasello's (e.g., Tomasello, 1999) insight that culture transformed primate cognition into human conscious thought. Humankind's primate ancestors presumably had phenomenal awareness and social life. When new brain structures led to gestural communication, hominids began to share information socially, which is one foundation of culture. That became central to humankind's biological strategy for survival and reproduction (Baumeister, 2005). Once vital information was held collectively, natural selection would favor individuals who were best able to access this information.

Physical evolution then changed course to facilitate social and cultural interaction. Corballis (2009) noted, for example, that the human vocal apparatus (the descended larynx) increases the risk of choking, as compared to that of other primates-but presumably this disadvantage was offset by being able to talk. Certainly in any human group with speech, talkers will survive and reproduce better than grunters. Likewise, human hearing seems adapted for speech. As any dog owner knows, dogs can hear many sounds that humans cannot, but they cannot distinguish as well among similar words (e.g., glad/bad/mad). Like all sensory systems, the ears must trade off detection (hearing a wide range of sounds) against resolution (hearing some sounds precisely). Most animal sense organs favor detection. Human evolution, however, appears to have reversed the general pattern, sacrificing detection in favor of resolution. The disadvantage of being unable to hear some sounds was presumably offset by the advantage of being able to distinguish among similarsounding words. Vocal imitation and word-learning capacity also appear to be much more extensively developed in humans than in most other species, and they likely contributed to human language learning (Hauser et al., 2002). In short, once the selection environment contained culture, being able to talk, hear, and learn words became vital to the survival and reproductive success of individuals.

The implications for consciousness theory are that phenomenal awareness was refined by selection factors favoring speech. A person can express only one idea at a time, whether by gesture or speech, despite having a brain with potentially many parallel cognitions. Thus, a new version arose of the dilemma that Morsella (2005) says phenomenal awareness is suited for: Parallel cognitions and conflicting impulses had to be resolved to produce coordinated single actions involving muscles moving bones. Early social communication depended on having muscles move the arms, and later, the focus shifted to the speaking apparatus. Conscious thought was needed for understanding the speech of others and producing one's own speech. Silent conscious thought was likely a by-product of this.

The link between conscious thought and talking may be relevant even to the shortcomings of conscious thought. The errors and oversights to which the single conscious mind is prone-including the ones detailed in the five critiques above-can often be corrected as people say what they think and get social feedback from others. If conscious thought is for sharing information with the cultural group, then the occasional mistakes of individual, private thought do not constitute a sweeping indictment of its value.

\section{Human Consciousness as Constructing Sequential Thought}

The third benefit of the brain making input into itself comes from advantages that emerge from combining information via conscious thought. The core of this argument is that conscious thinking can produce some useful kinds of information processing-ones that cannot be produced without consciousness. It is clear, however, that simple minds (animal and unconscious human) can learn many concepts and patterns and produce associations. For conscious thought to add anything, it is most likely in the special and meaningful combinations of concepts.

The idea that conscious thought has special capabilities may seem counterintuitive. Its limited capacity has been noted (e.g., Baars, 1997), and some critics of consciousness have pointed out that the processing capacity of the unconscious mind far exceeds what consciousness can do (Dijksterhuis \& Nordgren, 2006). Yet the unconscious is mainly powerful for handling learned or hardwired formulas, such as those that translate light into a navigable path or sound into the voice of a familiar friend. Novel processing of information (e.g., following a friend's driving directions) may require conscious thought. Even though consciousness cannot process as much information at once as the unconscious, it may be superior in processing information in new ways.

In our view, human consciousness is best understood as the place where the unconscious mind creates and processes meaningful sequences of thought. This provides a good fit to the empirical evidence for the benefits of conscious thinking. In this section, we consider several forms of sequential processing. Each one enables the processing of novel content. They also depend heavily on social interactions and a cultural environment. Later, we discuss how they provide the basis for understanding mental simulations of nonpresent events (including possible and counterfactual ones).

\section{Speech and Sentences}

We begin with language because the previous section suggested that evolutionary advances in conscious thought were driven by the demands and opportunities presented by living in a cultural environment where language was used to share information. The grunts and barks used by many animals to communicate are at best tantamount to communicating with single words, and even that interpretation may overstate the case (Hauser et al., 2002). In contrast, when humans began to use the sequencing of words (or gestures) to create meaningful combinations, the unique power of language began to be realized and made useful. Crucially, meaning depends partly on the sequencing of words, so that "Ann hits Bill" means something quite different from "Bill hits Ann."

Multiword speech is an important and basic form of sequential thought. Many thinkers have grappled with and puzzled over how language and conscious thought are related. Confusion arises because many nonhuman animals are able to understand and respond to words, thereby tempting some observers to count them as language users. However, almost invariably, these are limited to single words. At best, a few of the most intelligent individual chimps occasionally seem to understand once in a while that 
different sequences of signs convey different meanings. All normal humans understand this from childhood and spontaneously produce many different sentences every day.

The linguistic powers of the human unconscious likewise seem limited to single words. Priming studies have largely failed to get nonconscious responses to even two-word phrases (Draine, 1997; Greenwald \& Liu, 1985). Even negation, which combines a concept with a symbol that conveys negation, seems to elude the nonconscious mind (Deutsch, Gawronski, \& Strack, 2006). In dichotic listening tasks, people do process and respond to single words in the unattended channel—but not to sentences (Mackay, 1973).

In short, the unconscious mind can handle single words and concepts, but it needs conscious thought to take in sentences. Hence, again, conscious thought probably developed to enable the mind to use and benefit from the full powers of language and information. People can access the universe of possible ideas, including sentence and paragraph ideas, as well as narratives, but only by virtue of conscious thought.

Language is obviously learned from one's social environment. All known cultures use language, so any human growing up in a cultural environment encounters language and masters it as a key means for living in that environment. The cross-cultural universality of language is at least partly attributable to its immense benefits for transferring information from one person to another. These benefits include advancing social learning, facilitating social interaction, and coordinating effort on complex group tasks $(\mathrm{H}$. Clark, 1996). It is axiomatic that one does not have language alone, and so, language is inherently social, indeed cultural.

Carruthers (2002) concluded from research with brain lesions and other sources that thought without language is clearly possible but that language is generally needed to fill the brain with information to think about. Without language, thought would be quite limited. Thus, as proposed by Tomasello (1999), participation in culture via language was the essential step that transformed the primate brain's capabilities into the far-reaching power of human thought.

The difference between single-word communications, which many animals have, and multiword or sentence communications, which are pretty much unique to humans, is profound. First, sentences can convey vastly, literally infinitely more information than single words. For a species that uses the social sharing of information as a central part of its biological strategy, sentences are invaluable. Second, they create the possibility for new kinds of thought that are based on sequential steps, such as logical reasoning and mathematical problem solving (see next subsections).

Third, sentences introduce a level of flexibility that fosters the capacity for novel content, and this proves important to our subsequent discussion of conscious simulations. Words have fixed meanings, and so, single-word communications are rigid: The single-word commands known to dogs, for example, have specific and constant meanings. In contrast, when words are used to make sentences, they can produce many different meanings. The meaning is created partly out of the way the words are combined. Put another way, the mind that speaks a single word expresses a preset and rigidly stable idea-whereas the mind that speaks a sentence produces a novel, potentially unique meaning. Sentences can express ideas precisely suited to the unique current situation, and so, sentences are more flexible than single-word utterances and can express ideas that single words cannot.

\section{Counting and Quantification}

Counting constitutes sequential thought, insofar as one moves from one number to the next according to rules. Arithmetic is likewise sequential, as one combines numbers in various ways leading to different numbers. The rules of counting and arithmetic are objective rather than invented, but still, most people learn them from the social environment rather than discovering them on their own. Moreover, as with words and sentences, quantitative reasoning introduces the possibility of creating novel and flexible content from a fixed set of constants. As one simple illustration, the ten numerals ( 0 to 9 ) can be used to represent a literally infinite variety of different numbers. Human children seem to grasp this easily once they have learned a few numbers. Animals do not, and indeed, even chimpanzees can at best be laboriously taught to distinguish between a few basic integers-and still, each new integer is as difficult to learn as the last, indicating that the animals do not grasp the idea of the number system (Hauser et al., 2002).

As with language and reasoning, one can become confused about whether quantitative reasoning depends on conscious thought, insofar as the unconscious seems to know arithmetic. Presented with the problem " $7 \times 5$," most adult minds instantly have the thought " 35 " as an automatic response. As Winkielman and Schooler (2008) pointed out, however, nobody seriously believes the unconscious actually carries out the calculation, such as by counting the sum of seven rows of fives. Rather, arithmetic tables are overlearned by means of explicit repetition and rote memory during childhood, so that the association becomes automatic. The automatic response is thus the result of earlier conscious processing.

The Cognitive Reflection Test developed by Frederick (2005) reveals the difference. In a typical problem, Charlie purchased a bat and a ball for $\$ 1.10$, and the bat cost a dollar more than the ball; the question is how much did the ball cost? The automatic system typically furnishes the incorrect answer of 10 cents, which has to be overridden by the conscious system that can calculate the correct answer. When the conscious mind is preoccupied, the likelihood of the automatic but wrong answer increases.

Quantification is highly useful in culture. Resource management, planning, and decision making, including in military and hunting contexts, all benefit from quantification. Cooking for a large group can be substantially improved by quantification, both in terms of getting the right proportions of ingredients for recipes and in terms of making the right amount so as to avoid waste and shortfalls. Above all, economic marketplaces and transactions depend inevitably on quantification (e.g., how many fish for so much grain; see Weatherford, 1997), and economic trade is one of the most important driving forces in culture and cultural progress.

\section{Logical Reasoning}

Logical reasoning is an important and influential form of sequential thought. It enables the thinker to proceed from one idea to another according to rules. Logical reasoning greatly increases the practical value of information. It enables the mind to realize new truths based on information it possesses. Thus, one bit of infor- 
mational input can lead to multiple useful conclusions. In group tasks and group problem solving, logical reasoning enables the best and most pragmatically useful view to be implemented, as opposed to simply the view advocated by the physically dominant male.

The rules of reasoning (like those of arithmetic) are objectively true, but in practice, most people probably learn from their social environment how to reason rather than discovering the principles on their own. Effective reasoning is taught both informally (by parents and friends) and formally (in schools and universities). The rules of inference that dictate what sorts of conclusions may be drawn from what evidence do vary somewhat (even among academic disciplines) and, hence, probably also from one cultural group to another. What is rational and logical is not entirely an objective matter and depends to some degree on social consensus (Shiffrin, 2008). These socially shared agreements extend the power of reasoning, such as by defining what counts as proof (e.g., scriptural text, legal precedent, quantified observation).

Indeed, reasoning may be even more fundamentally social than merely dependent on social learning. The influential philosopher Davidson (1982, p. 327) concluded on conceptual grounds that "rationality is a social trait. Only communicators have it." His derivation invoked the nature of belief as possibly true or false, which requires a social context in which multiple individuals know that they have different beliefs that cannot be simultaneously true. Even if one were to dispute Davidson's conclusion that rationality inherently and inevitably requires a social context, it is almost certainly correct empirically that people learn the relativity of beliefs by encountering others who believe differently and from there learn the value of logic for resolving disagreements.

Evidence has accumulated that logical reasoning is the province of conscious thought. Lieberman, Gaunt, Gilbert, and Trope (2002) concluded from assorted evidence that automatic, nonconscious systems do not properly engage in logical reasoning (see also Smith \& DeCoster, 1999, 2000). Evidence in favor of the logical capabilities of the unconscious, such as that offered by Lee and Ariely (2006), depends on inferring logicalness from consistency of responses (e.g., transitivity), but all this points more toward consistent preferences than actual reasoning. De Neys (2006) showed substantial decrements in logical reasoning as a function of cognitive load, except when shortcuts were available that enabled the mind to bypass the actual reasoning process.

Direct tests of the hypothesis that conscious thought is needed for reasoning were reported by DeWall, Baumeister, and Masicampo (2008). When conscious processes were hampered by cognitive load, the rate of correct answers to logic problems dropped to chance levels, equivalent to guessing. Conversely, increasing conscious involvement in reasoning (by setting conscious goals and telling participants that they would have to explain their answers later) improved performance. The corresponding manipulations of unconscious processes had no effect. Priming the idea of logic activated the motivation and even increased attempts to appear logical, but correct answers did not increase. An unconscious cognitive load manipulation had no detrimental effect on reasoning performance. Thus, the manipulations of conscious processes affected logical reasoning, whereas manipulations of unconscious processes had no effect on it.

The misleading appearance of unconscious reasoning stems from learning and efforts to estimate. In one problem used by
DeWall et al. (2008; adapted from Markovits \& Nantel, 1989), participants were instructed to evaluate whether the following argument is logically valid: All mammals can walk; whales are mammals; therefore, whales can walk. Everyone knows the conclusion is wrong, but that is because of faulty premise- the argument is perfectly valid in purely logical terms. Priming the concept of logic increased the unconscious motivation to be logical, resulting in an increased number of wrong responses to such items (i.e., saying the argument was invalid). Unconscious processes respond to the motivational cue to be logical by using all they can do, such as consulting memory knowledge about whether whales can walk.

Further evidence of the social and beneficial nature of conscious thought came from a meta-analysis by Fox et al. (2009). Whereas merely thinking aloud did not alter performance at solving problems, telling participants to explain their efforts led to significant improvements in performance across studies. Demanding explanations forces the participant to think rigorously and interpersonally about the problem, and the evidence indicates that these thoughts improve the outcome.

Like sentences and quantitative thought, logical reasoning creates the possibility for mental acts to reach novel content. Reasoning leads from one idea to a different idea, even if no prior association exists and there was no preexisting association between the premise and the conclusion.

\section{Causality}

Causal interpretation is another form of sequential thought. It moves beyond association because it is unidirectional (cause produces effect) and conforms to rules that privilege one result over other possible associations (see Satpute et al., 2005). Knowing a causal relationship enables the person to predict what will happen under particular circumstances. As with the previous forms of sequential thought, there is some objective reality to causation, but by and large, people probably learn most causal thinking from other people rather than discovering principles of causation for themselves.

Causal understanding and advanced quantitative reasoning are "uniquely human and universally human" (Tomasello, 1999, p. 188). Primates can master causality in the sense of seeing that one physical event causes another, and they can build on that understanding to make and use tools, but Tomasello (1999) concluded that they lack the human understanding of causality as a matter of invisible forces that connect visible events. (In the same way, he concluded that primates have elaborate social interactions and relationship skills but lack the understanding of others' behavior as caused by inner states such as intentions.) That conclusion is highly congenial to the view that conscious thought is needed to use these forms of understanding.

Indeed, when Hauser et al. (2002) tested the mental abilities of human children, chimpanzees, and orangutans, causal reasoning was the lone exception to the general pattern that social cognition (as they defined it) accounted for all the differences between the humans and the other primates. This led them to speculate that causal understanding might well have a social dimension, insofar as both social cognition and causality entail understanding events as driven by invisible forces. They also pointed out that even if the differences between humans and other primates are best described in terms of understanding invisible forces, this could have evolved 
for social cognition (understanding conspecifics) and then become generalized to inanimate objects in the physical environment.

The social learning of causation is evident in collectively shared false beliefs about causation, including supernatural causation. Although it would be impolitic to suggest that all religions subscribe to false beliefs about causation, most religious people regard other religions as containing falsehoods, so we may safely suggest that many religious beliefs are false. Religions commonly include beliefs about supernatural causation, ranging from how the world was created through the actions or misadventures of supernatural beings all the way to interpreting specific misfortunes (e.g., illness, failure) as caused by divine intervention (Berger, 1967; Eliade, 1978, 1982). Hardly anyone today believes that sacrificing animals is an effective means of causing improvements in weather or military outcomes, but such beliefs were once commonplace. That groups of people would share false beliefs about causality (and then together stop) is one sign that people learn them from other people.

\section{Narratives}

Narratives constitute an important form of human speech. Abstract principles may or may not be inferred and articulated, but much information is retained and transmitted in narrative form. Narrative thought also enables people to understand their behavior as part of a meaningful story and to alter it on that basis.

We hypothesize that narrative thought requires conscious processing, at least to be fully effective. Narratives essentially build on the four forms of sequential thought we have already mentioned. That is, a viable narrative is rendered in multiword speech, makes logical sense, and is causally plausible. Quantification is less obviously relevant to many stories; still, errors of quantification can damage the credibility of stories.

The capacity for conscious thought to construct meaningful sequences of events is exemplified by the human tendency to organize experiences into structured narratives. Narrative stories enable people to link events and actions causally over time (e.g., Graesser, Singer, \& Trabasso, 1994; Mar, 2004), and they have been described as the fundamental constituent of human thought and communication (Bruner, 2002; Schank \& Abelson, 1995). Indeed, the tendency to string events together into a narrative appears to be universal (Barthes, 1977) and automatic (Wyer, Adaval, \& Colcombe, 2002). Moreover, the conscious rehearsal of discrete events appears to play a crucial role in developing causal links between them (Wegner, Quillian, \& Houston, 1996). Thus, meaningful sequences of events are a major element of human mental experience, and conscious thought seems instrumental to their formation. Even more relevant to the current discussion is whether these meaningful sequences are at all helpful for acquiring information about the world. Costabile and Klein (2008) argued that, though cognitively expensive, the structuring of experience into meaningful narratives is helpful for understanding past social events and for making predictions about the future. Meaningful narratives are also instrumental in shared learning. People often transfer narrative lessons to others via gossip (Baumeister, Zhang, $\&$ Vohs, 2004) or the telling of fiction (Mar \& Oatley, 2008). These findings are consistent with the simulation hypothesis. Conscious thought creates meaningful sequences of events, and in so doing, it provides people with new and helpful information on which to act.

Constructing narratives is thus an important form of human thought. Actual events can be understood and told as narratives. However, perhaps even more important, once the mental capacity to construct narratives exists, it can be used in ways that depart from direct experience. In essence, the mind acquires the ability to simulate possible events rather than just perceiving and understanding actual ones. This capacity for simulation constitutes a main focus of our analysis, and we return to it shortly.

\section{Implications}

We have argued that the special powers and advantages of conscious thought involve sequencing concepts. Some sequences are favored over others (e.g., there are right vs. wrong answers to arithmetic and logic problems) based on rules that are generally learned from the social group. This allows conclusions to be reached that would not be apparent from simply having the assorted information scattered in memory. Logical reasoning, costbenefit analyses, and mathematical calculations all involve use of culturally shared rules to reach novel conclusions from information one already has. For example, when one has a plane to catch tomorrow, one typically engages in a simulation that calculates backward from the plane's takeoff time, allowing for airport procedures, the trip to the airport, and perhaps the hotel checkout before that, so one knows at what time to commence the sequence of acts. All the information used for this simulation is already in the mind, so conducting the simulation does not bring in new information from the environment. Rather, it privileges some forms of association (e.g., culturally shared rules about arithmetic that relate the times of multiple steps to the scheduled departure time) over others (e.g., what times one remembers leaving for the airport on one's previous journeys). These simulations work remarkably well in enabling people to be on time for their flights without having to spend many extra hours at the airport. We invite skeptics who dismiss the value of conscious thought to skip the conscious simulation when they travel and see how well they fare by relying solely on unconscious thought and automatic responses. The general conclusion is that conscious thought uses information to produce novel conclusions and insights, which the nonconscious executive can then use to improve the effectiveness of its actions.

Conscious thought enables the processing of information from culture so that the human animal can operate within it. Thus, conscious thought, the rules that it uses, and the ultimate output are all social and cultural phenomena. On its surface, the previous airport example may seem like a very nonsocial form of thinking and behaving. Yet every step of the process requires coordination with others. For one, arithmetical rules are a cultural phenomenon: The passenger was taught by others how to subtract and how to use clock time. Furthermore, the very act of setting a flight departure time is dependent on culturally shared information. It is contingent on the fact that each person involved, including the pilots, flight attendants, desk attendants, and the passengers, shares the same concept of time (and that they will all coordinate their behavior on that basis, even if they are strangers). Clock time is itself socially relative: Prior to mass transit (trains), each town constituted its own time zone, based on the cultural convention of setting noon at when the sun was directly overhead. Moreover, a timely departure 
requires that each person is using the same set of mathematical rules to ensure that he or she arrives at the appropriate hour. Conscious thought thus manipulates information from memory using rules learned from and shared within culture.

All of this points toward conscious thought as conducting simulations that go beyond present sensory input and direct control of immediate actions. The next section elaborates this core function of conscious thought.

\section{Conscious Thoughts as Simulations}

The argument thus far has been that conscious thought represents the workspace or theater where the automatic mind constructs meaningful sequences of thought. These conscious thoughts are several steps removed from direct sensory input and direct behavioral output. They can, however, be useful for processing information that is highly complex, such as information about the social environment.

In this section, we carry this argument a step farther and propose that many of the most useful and important functions of conscious thought are not for dealing with the immediate here and now at all. Rather, they are for simulating events, especially ones away from the immediate present.

\section{Consciousness Is Constructed}

We have suggested that conscious thought is best suited not for observing and controlling events but rather for simulating events, which is to say imagining possible events. After all, the point of a simulation is to imitate some possible reality to explore and test it. Thus, consciousness constructs possible realities rather than reliably reveals definite facts. Many of the criticisms we have mentioned, including those by Gazzaniga, Wegner, and Nisbett and Wilson, amount to showing just this: What occurs in consciousness is not necessarily correct but rather is a constructed representation of what is probably right. Even the subjective feelings of doing may be constructed simulations, as indicated by small errors even there (Haggard \& Cole, 2007; Wegner, 2002).

By emphasizing construction, we move beyond theories that treat consciousness mainly as a process of selection (see also Shanon, 2001). Baars (1997, 2003) described consciousness as featuring a spotlight that may shine on any of a number of items in a centrally visible workspace. Similarly, Crick (1984) described consciousness as a searchlight, the focus of which is vied for by competing coalitions in the brain (Crick \& Koch, 2003). These models imply that the contents of consciousness have already been waiting somewhere in the brain and that the job of consciousness is to put those contents in touch with other mental contents.

Although the searchlight metaphor is useful, our theory emphasizes an additional feature of conscious thought. Conscious thought does not simply illuminate some pieces of information in the mind, any more than speech merely reads off sentences that are already fully formed prior to saying the first word. Rather, conscious thinking and speech involve a process of actively combining concepts to make something that may have additional, unforeseen, newly emergent properties. In that sense, the contents of conscious thought are constructed, and this is essential to its function. Yes, the information from which the conscious simulations are made is already present in the mind and brain, so conscious thought itself does not import any new information from the world. However, by combining this information into a constructed simulation, conscious thought can create something that differs from the assorted separate pieces of information it already had. Logical reasoning is a good example of this: One starts with premises and, without importing new information but by rulebased thinking, produces a new conclusion. Likewise, by rehearsing a plan mentally, one can evaluate whether it is a good idea and possibly improve it, even without obtaining new information from the environment. (Moreover, if one formulates the plan to tell it to others, one can then receive social input to improve it.)

The constructive aspect of conscious thought may be an extension of the constructive processes that underlie phenomenal awareness. Phenomenal awareness is not just seeing what is there. Rather, the conscious experience has already had the benefit of extensive interpretive work, drawing on memory and knowledge to react appropriately to the incoming sensory data (Edelman, 2004). Research on vision has completely demolished the simple-minded view that what one consciously sees is a result of the eyes conveying information directly to a display somewhere in the brain. Information is disassembled as it comes in, is processed separately by multiple modules, and then is reassembled into a coherent picture. Ungerleider and Mishkin (1982; Mishkin, Ungerleider, \& Macko, 1983) have shown that the two basic perceptual tasks of determining what one sees and where it is are performed separately in the brain, with that information later recombined.

Likewise, feature migration effects (e.g., Treisman, 1988; Treisman \& Gelade, 1980; Treisman \& Schmidt, 1982) occur because features are processed separately and then recombined, thereby introducing the possibility of error. For example, someone who sees red triangles and green squares may mistakenly recall having seen a red square, because shape and color are processed separately, so that the conscious experience is constructed by combining results of these separate processes. (Much of this occurs well before the conscious image is experienced.)

The extensive literature on perceptual illusions and distortions provides further evidence that the conscious image is the result of a constructive process. The mental image is created from multiple pieces of information, and it is therefore susceptible to relying inappropriately on some cues. The facts that the moon seems larger when near the horizon and that a line with outward flanking arrows is seen as longer than a line with inward flanking arrows (in the Müller-Lyer illusion) indicate that the mind relied on cues other than the incoming light waves to construct the image. Thus, the mind is actively creating sensations, not merely attending to or noticing them. As Humphrey (2006) put it, sensations are not something that happens to a person but rather are something that a person does. Extending this view to the level of conscious thought, we suggest that one does not simply gain access to information (e.g., by allowing the information to come into focus) but instead creates something new with that information.

Constructive simulation is also what occurs in human communication via sentences, which is how people get information from their social group and culture. This is how speech operates, but it can perhaps be even better seen in an example of text messaging by cellular phone. The communicator has a meaningful idea in his conscious mind. To communicate it, he relies on automatic, nonconscious processes that transform the idea into a sequence of words, which are then rendered in individual letters, which are 
executed by thumb movements, which in turn are caused by nerve cell firings. The conscious meaning cannot be discerned in the individual nerve cell firings or the thumb movements, since, after all, the same movements express many other ideas. (Even the spelling and the individual words do not capture the meaning, which is encoded only in their sequence.) The receiver's eyes receive the sensory input of light patterns, which are converted by nonconscious processes into letters and words and, ultimately, fully reconstructed in her mind as sentences. From this, she understands the same meaning (one hopes, at least) that he intended. Thus, the exchange of information is carried out by the unconscious processes of the two parties, enabling an idea to transfer from his conscious mind eventually to hers. Conscious thought is for constructing and simulating the idea, not for carrying out the exchange.

Episodic memory, which, like conscious thought, is widely considered to be uniquely human (e.g., Tulving, 2002, 2005), has long been acknowledged as a constructive process. Retrieval does not involve shining a light on some dusty old episode that has been waiting intact in the attic of the brain. Instead, retrieval involves recreating a past experience given one's expectations about what probably occurred (Bartlett, 1932).

Researchers have long pointed to the puzzling fact that memories change as further evidence of memorial construction. Initially, it was assumed that memories change according to a temporary process of consolidating the initial input, which already points to construction. More recent work (e.g., Hupbach, Gomez, Hardt, \& Nadel, 2007) has shown that memories continue to change over long periods of time, as long as they are recalled periodically. The implication is that each time an event is remembered, it is reconstructed from specific facts remembered about the event but perhaps is also informed by other information and motivations. Moreover, each replaying of the event adds a new version to memory. As this information changes, so does the memory.

Thus, memory constructs and simulates past events, rather than replaying them literally. The implications of this have been developed in recent writings by Suddendorf and colleagues (e.g., Suddendorf \& Busby, 2003, 2005; Suddendorf \& Corballis, 1997, 2007). Nondeclarative memory, such as retaining a physical skill or changing behavior based on reinforcement history, is obviously widespread in many animals and can easily occur without conscious thought. If anything is uniquely human, then, it is the capacity to reconstruct past events in one's mind as vivid sequences. Yet what good is that? Suddendorf (e.g., Suddendorf, 2006) has emphasized the point that remembering the past is not itself of any use, and its adaptive value can only be found in flexible application to present and future events. The fact that memories are constructed and changing, rather than exact replays of what originally happened, is thus converted from a weakness to a strength: The reconstruction of the past event can be altered to suit present and future concerns.

Even more important, Suddendorf and Corballis (1997, 2007) proposed that recollection and foresight are two aspects of the same function, which they called mental time travel. As evidence, they noted that the abilities to predict the future and to recall the past emerge at about the same time in child development (Busby $\&$ Suddendorf, 2005). Furthermore, memory and foresight suffer intercorrelated impairments in various cases of brain damage or mental affliction, and indeed, similar patterns of brain activity are found with remembering past events as with predicting future ones (Suddendorf \& Corballis, 1997). Given that foresight has huge adaptive benefits, whereas recall without application to present or future cannot have much if any benefit, one implication is that episodic memory may be a by-product of the capacity to simulate future events. It is quite plausible that these simulations are central to human consciousness. There is still no convincing evidence that nonhuman animals can engage in deliberate mental time travel to either past or future (Suddendorf \& Corballis, 2007).

Putting these together, it seems that humans or almost-humans evolved a new kind of memory capacity, with vivid recreation of past events, yet constructed rather than reliably replayed. Moreover, this was probably a side effect of some other capacity because remembering has much less adaptive utility than foresight and other forms of thought. Transcendence (i.e., responding to something beyond the here and now) is common to both memory and foresight. Recall often involves replaying an episode counterfactually, which requires some of the same brain structures that enable thoughts of the future (Smallwood, Franklin, Chin, Handy, $\&$ Schooler, 2009). The construction of sequential thought is also essential to both declarative memory and intelligent forecasting. These all seem to be tied to conscious thought, so our hypothesis is that declarative memory is likely a side effect or by-product of the evolution of conscious thought, which evolved to do things for which the flaws of memory are virtues - specifically, constructive simulation. That is, construction following rules and influenced by current motivations and new information makes memory less accurate over time, but it improves foresight. Foresight would be less effective if the mind clung rigidly to previous knowledge and information than if it flexibly adapted ideas to new circumstances and current concerns.

Many memory researchers are coming round to embrace the view that episodic memory may be less designed for recording the past than for projecting into the future (see Buckner \& Carroll, 2007; Schacter \& Addis, 2007), as indicated by its constructive nature. Further evidence comes from research on mind wandering, which has shown that wandering to thoughts of the future is twice as frequent as to thoughts of the past (Smallwood, Nind, \& O'Connor, 2009). From our view, the construction process allows the mind to create simulations of hypothetical events. Conscious thoughts are simulations that are meant to provide input into what might or could happen, rather than what definitely did happen at some point in the past.

\section{Constructing Serial Sequences}

Some writings on conscious thought have depicted it as an inner blackboard (Baars, 1988). Although such a metaphor has value, it misses the important quality of continuous movement and of connecting some events to others, which is why we prefer the metaphor of a film. The contents of conscious thought seem always to be moving so that one's conscious thoughts move readily from the present to impending outcomes. Short-term (or working) memory is one of the cognitive processes most closely linked to conscious thought, and continuous movement appears to be one of its features. If short-term memory were simply a storage facility like long-term memory, one ought seemingly to be able to put information into it (seven plus or minus two bits of information, according to Miller, 1956) and then retrieve it later on. 
Instead, however, it seems necessary to keep repeating and rehearsing information, if it is to be retrieved (Baddeley \& Hitch, 1974). Glenberg (1997) proposed that, because short-term memory does not resemble a static store but rather a movement along a trajectory, the purpose of short-term memory may be to simulate possible actions. Insofar as conscious thought is linked to shortterm memory, then it too may be unable to sit still.

Conscious thought constructs meaningful sequences so that each new event or image follows from previous ones. The film analogy is helpful insofar as there are two different ways of making a film. In the standard style, the image is made from what the camera sees: Each frame in the film is directly produced from the camera input. In animation, however, each frame of the film is made by starting with a copy of the previous frame and then altering the drawing as needed. For camera-style filming, the process requires equipment that specializes in converting input into image. For animation, the process emphasizes adapting a previous image to make the next one, and so, the hardware is designed to make images out of other images. Consciousness, in this view, resembles animation more than camera filming. New input from the senses is used to update the existing image more than to create a thoroughly new one. Phenomenal awareness may operate in that way, and conscious thought adds a greater capacity for building on the contents of consciousness irrespective of new input. A person can thus imagine a potential consequence even if there is no direct evidence for it in the current sensory input.

The development or evolution of consciousness presumably always involved some aspect of construction. Early on, construction meant integrating incoming sensory information, such as merging input from the two eyes. Stored knowledge is accessed to identify stimuli, so that the image constructed in phenomenal awareness is known and understood to some degree. Presumably, the identifying process is not repeated with each new light wave that strikes the eye: Instead, once something has been identified, it retains its identity in phenomenal awareness. Thus, a simple version of phenomenal awareness is like a small movie about the immediate present that the animal's mind constructs from sensory input.

The inner film-making apparatus is evident during sleep. There is little incoming information, yet consciousness is still there and capable of work. Having no actual sensory input to use, it instead works from inner stimuli, such as memory or even random neural activity (Seligman \& Yellin, 1987). Dreams are the result.

Dreams furnish a plausible intermediate step on the road to the full construction that is conscious thought. They represent an important advance insofar as they create a kind of inner sequence of experience without direct input from the current stimulus environment. In dreams, the mind creates experiences that are so lifelike as to convince a person that they are real (Foulkes, 1985). Such virtual experiences provide an ideal medium through which to simulate events for the benefit of the self. Indeed, threat simulation theory (Revonsuo, 2000) proposes that the function of dreams is to rehearse threatening events for the purpose of learning how to cope with them in one's waking life. In support of that view, a large majority of people's dreams involve negative rather than positive emotions (Hall \& Van De Castle, 1966). Thus, dreams are more often used for dealing with undesirable events and consequences than the seemingly less useful rehearsal of positive ones. Moreover, a majority of people's negative dreams involve threatening social interactions (Hall, 1955). As may be the case with conscious thought, dreams enable mental simulations and rehearsals, often for the benefit of improving one's interactions with other people.

The evolutionary record also supports the idea of dreaming as an intermediate step toward conscious thought. It is generally assumed that animals dream but do not engage in conscious thought in the fully human sense. The content of animal dreams remains unknown, given the lack of verbal self-reports, but muscle movements suggest hunting, fighting, and eating are involved. There is also some evidence that dreaming is beneficial for learning via reprocessing of memories (Cartwright \& Lamberg, 1992; Stickgold, Hobson, Fosse, \& Fosse, 2001). This is important because it suggests that nature was finding uses for the inner sequencemaking apparatus apart from processing currently incoming information. In humans, at least, dreams are also overwhelmingly social, in the sense that they depict interpersonal events and relationships (e.g., Snyder, 1970).

The full power of human consciousness therefore arose from using the mental capacity for constructing sequential thoughts to conduct simulations during wakefulness, without relying on sensory input. Very possibly, this proceeded by dreaming while awake, including daydreaming. For this to happen, a variety of further design problems would need to be solved. After all, it is safe for the mind to wander during sleep-dreaming because the subjective experience will not interfere with ongoing task performance, insofar as the sleeper is not performing tasks. An animal sleeping in the forest can safely dream about running across a field, but if the animal mentally simulates running through a field while it is awake and actually running through a forest, it would crash into trees. Thus, the conscious mind must be able to maintain recognition at some level that it is simulating nonpresent realities while continuing to monitor current input. The driver may be concentrating on singing along with the radio or arguing with a passenger but can still step on the brake if the light ahead turns red.

Another term, mind wandering, has been recognized as a source of performance decrements, which it certainly is for tasks that require sustained focus on the present (Smallwood \& Schooler, 2006). Yet one should perhaps not be surprised that mind wandering occurs. If, as we suggest, the great advances in the power of conscious thought served precisely these functions of enabling the human mind to work on social problem solving away from the immediate present, then it is thoroughly natural for the human mind to wander (Mason et al., 2007). Prolonged focus on uninspiring tasks, such as reading a statistics textbook, would be the unnatural and, hence, difficult achievement (e.g., Schooler, Reichle, \& Halpern, 2004). Consistent with that argument, the mind wanders most when mental resources are low (Sayette, Reichle, \& Schooler, 2009; Sayette, Schooler, \& Reichle, in press). Moreover, when conscious thoughts do wander spontaneously, it is thoughts of the past and future — not of the present - that win out.

Many dual process theories of mind have likewise emphasized that the processes linked to conscious thought involve constructing sequences. Shiffrin and Schneider (1977) contended that automatic processes operate in parallel, whereas the more conscious processes operate in serial. Serial could mean simply one thing after another, but a more profound conception of seriality would emphasize that some sequences are more proper than others- that is, the sequence is not random but rather based on meaningful con- 
nections. Some sequences are privileged over others, and mature conscious thought follows these rules. In principle, sequencing rules may be acquired on one's own, but also they are susceptible to learning, such as when schoolchildren memorize multiplication tables, develop schemas over time, learn how to predict what follows what, and internalize shared expectations from the culture. Whatever the source, conscious thought seems to connect consecutive events by specific rules and patterns.

\section{Conscious Versus Unconscious Simulations}

The view that conscious thought is simulation becomes less radical if one embraces the view associated with recent trends in so-called embodied or grounded cognition and modal cognition (see Barsalou, 2008). Essentially, in these views, all cognition, starting with perception, is simulation.

Many of the most basic forms of thought can be understood as pattern completion (Barsalou, Niedenthal, Barbey, \& Ruppert, 2003). Once a pattern is known to the mind and part of the pattern is recognized, the remainder may be automatically activated: If $\mathrm{X}$, then Y. The simple expectancies on which animal learning is based (see Rescorla \& Wagner, 1972) illustrate such pattern-completing processes. There is no basis for assuming that such cognitions require conscious thought in the human sense-or indeed any awareness at all. However, if even unconscious thoughts consist of simulations, then to understand consciousness, one must ask what differentiates conscious simulations from unconscious ones.

Why do some simulations become conscious? In particular, what produces the presumably advanced and uniquely human simulations in conscious thought? To answer, it is again useful to invoke Morsella's (2005) insight that consciousness resolves conflicting prescriptions for skeletal muscle movements arising from parallel processes. Simple phenomenal awareness may help many animals resolve basic conflicts in the immediate present, such as whether to flee or to continue eating. The simulations in human conscious thought, in contrast, may be useful for resolving conflicts arising from the demands of the complex social and cultural environment, such as whether to accept an offer of trade or of illicit pleasures.

Moreover, the simple pattern-completing simulations such as expectancies may be relatively automatic and inflexible. Considerably more work is required to conduct flexible simulations with novel content. Rehearsing tomorrow's negotiation may involve imagining several different things the other party might say and trying out various possible responses. Constructing multiple, alternative simulations of the same future event is undoubtedly useful for people but probably requires the capacities of conscious thought that we have already outlined, such as multiword speech, causality, quantification, and logical rationality. As we have proposed, the capacity to construct flexible sequences of thought with novel content was acquired to speak and understand sentences and is thus inherently social.

\section{From Simulation to Action}

Conscious thought processes provide one with access to nonpresent information, which can be used to recalibrate the automatic responses that guide behavior. Thus, conscious thought can influence behavior indirectly. In the terms of James's (1890) ideomotor theory, there is no command to act per se. Rather, the conscious image of some distal goal, if held in place long enough without drawing internal opposition, triggers the unconscious mechanisms that are required to pursue it.

Here, we describe two processes through which conscious thoughts and simulations can translate into altered future actions. First, conscious thought creates simulated experiences to which one can form novel attitudes and associations, and these can translate into new, behavioral responses. Second, conscious thought can be used to favor some associations over others, such as when planning or acquiring information from others.

Altered associations through simulated experience. The contents of conscious awareness provide one with a reasonably accurate model of the world (Yates, 1985). Through simulations within that model, one can feel how joyful it would be to reach some long-term goal or how painful it would be to fail, and these simulation-induced feelings can influence the automatic associations that ultimately drive behavior. By simulating some event or experience, an individual can react to it without having to experience it directly.

For simulation to have such an influence on behavior, it must resemble actual experience. Hesslow (2002) provided a useful review of the neuroscientific evidence showing that conscious simulation fulfills this requirement. First, imagined perceptions and actions activate the same areas of the brain as actual perceptions and actions. Thus, the imagined act of throwing a baseball is nearly identical to the actual one in terms of the brain structures that it uses. Second, simulated perceptions mimic actual perceptions even for chains of events that have not been previously experienced. Thus, simulations make novel predictions at the perceptual level.

Conscious thought thus reconstructs experience. This idea is consistent with the theory of grounded cognition, which rejects the idea that amodal symbols are the medium of thought. It emphasizes instead that thought is composed of sensory (i.e., modal) simulations (Barsalou, 2008). People often assume that thinking about a task is quite separate from and different than doing it. Yet the mere act of thinking about an emotional concept requires recreating and reexperiencing that emotion (Niedenthal, Winkielman, Mondillon, \& Vermeulen, 2009). The overlap between thought and experience may explain why imagination of an event can lead to the false belief that it actually took place (Garry, Manning, Loftus, \& Sherman, 1996) or why people sometimes mistake dreaming for actual experience.

Conscious thought influences behavior indirectly by causing novel attitudes and associations to be formed toward simulated objects and events. This process is perhaps best exemplified by work on phobia formation in the absence of multiple traumatic actual encounters with the feared object (e.g., Bogels \& Zigterman, 2000; Ottaviani \& Beck, 1987). Phobias may thus develop and be sustained by involuntarily imagining traumatic encounters. If some object is simulated repeatedly as causing tragic or catastrophic events, then a strong aversion to that object may be formed. Of course, this same process applies to nonphobic responses as well. Through repeated mental simulations, people are able to cultivate a range of attitudes toward objects without ever encountering them. A traveler may learn to adore Barcelona before she has ever set foot there, and a history buff may develop a genuine hatred for some infamous figure whom he has never met. Thus, conscious 
thought may play a crucial role in forming attitudes to nonpresent stimuli. By simulating some object or event, a person can learn to like or dislike it without experiencing it in the flesh, and these attitudes can in turn influence future actions.

Simulations do not have to be perfectly accurate to be adaptive. According to the durability bias, people's predictions about the amount of distress they will feel in response to future misfortunes are often greatly exaggerated (Gilbert, Pinel, Wilson, Blumberg, \& Wheatley, 1998). Life tragedies - or even minor annoyancesusually turn out to be much less stressful than people anticipate. The prevailing view of such affective forecasting errors is that they are maladaptive (Dunn \& Laham, 2006). However, the function of simulations may not be to estimate the precise quantity of distress. Rather, their more simple purpose may be to foresee whether distress will be present. Regardless of the amount of distress, the motivational implication is the same: Avoid the behavior. If affective forecasts were turned around, such that distressing experiences were mistakenly anticipated as pleasurable ones, then that would pose a more serious threat to the functionality of mental simulations. As it stands, forecasting errors pertain to the quantity and not the quality of the anticipated experience.

Indeed, the tendency to exaggerate future displeasure may be not a miscalculation but rather a helpful and adaptive response (e.g., Haselton \& Buss, 2000). The capacity for conscious simulations to influence behavior may be bolstered by exaggerations of the severity of future feelings (Ainslie, 2007). Given that a mental simulation is only a watered-down version of actual experience, the boost in expectations about future pain may serve as additional motivation to avoid the negative consequences (e.g., defensive pessimism; Norem \& Cantor, 1986).

Incorporating novel information. Conscious thought can alter future behavior by favoring some associations over others. Making plans is one example. One is much more likely to follow through with an intention when one supplements it with a specific behavioral plan, often in the form of an "if ... then" statement (e.g., "If X happens, then I will do Y"; for a review, see Gollwitzer, 1999). Formulating such a plan creates a link between the anticipated cue (X) and the desired behavior (Y). As a result, perception of the cue causes the behavior to be executed automatically and without conscious guidance (Brandstätter, Lengfelder, $\&$ Gollwitzer, 2001). In essence, the conscious plan reprograms the automatic response. Moreover, simulations are essential to this process. One must be able to simulate the many possible plans of action, select one that is appropriate, and use that information to forge the novel association that manifests ultimately as an altered future response.

Information acquired from others can also be used to change automatic responses. For example, telling someone that a tone will be followed by a shock is sufficient to cause physiological arousal in response to the tone (Cook \& Harris, 1937). Likewise, a learned physiological response (e.g., through pairing of a stimulus with a shock) can be greatly reduced simply by telling the person that the usual consequence will no longer occur (Colgan, 1970). Thus, conscious thought can use information acquired from others to recalibrate automatic associations and so alter responses indirectly.

The power of conscious thought comes from its ability to provide the individual with helpful expectations and simulations about nonpresent events. As with any simulation process, conscious thought creates an internal version of reality so that it may be observed, and these observations affect later behavior. Plans that seem viable during internal simulations may be executed at a later time. Information acquired from others can be used to favor novel associations over old ones. Conscious thought may not directly produce behavior online, but it can alter behavior in indirect yet adaptive ways.

\section{How Simulations Facilitate Social and Cultural Life}

Central to the recent attacks on conscious thought has been the questioning of what special value it adds to the already effective and efficient automatic, nonconscious processing systems. As we have reviewed, researchers have generated considerable doubt as to whether conscious thought has much value for enhancing the ability to get information from the stimulus environment or for enhancing the ability to control action directly. Our analysis has therefore assigned quite different jobs to conscious thought.

Specifically, we emphasize how conscious thought enables the human animal to interact with the cultural system. To function in culture, the animal requires psychological traits that enable it to interact with the often invisible realities that comprise culture, such as moral values, social norms, honor codes, libel laws, rules of war, group mission objectives, legal technicalities, market economies, gossip, voting, negotiations, and paying on credit. In that perspective, the function of conscious thought is to enable the physical body to deal with the cultural system. Sure enough, those invisible realities consist of and require the sorts of meaningful sequences that conscious thought is apparently needed to process, including sentences and narratives, causation and responsibility, numbers, and, of course, logical rationality. In this section, we seek to make those conclusions more plausible by proposing how the capacity for simulating events other than the immediate here and now would be useful to cultural animals.

\section{Understanding Other People}

Human social life depends heavily on people being able to understand the mental states, emotions, intentions, and perspectives of other people. Direct sensory input provides only limited, indirect access to what another person is thinking or experiencing, so the ability to mentally simulate the inner states of others is crucial (Goldman, 1989; Gordon, 1986; Harris, 1991; Jeannerod, 2001; Tomasello, 1999; Tomasello et al., 2005). The closest thing to sensory input about the mental states of others comes from what they say - and it is arguable that language itself depends on so many shared assumptions and understandings that it presupposes theory of mind (i.e., the understanding that others have inner states comparable to one's own and the ability to imagine and empathize with the mental states and processes of other people). Indeed, the evidence from both developmental and evolutionary psychology seems to indicate that a primitive understanding of others' mental states precedes language and not the other way around (for a review, see Malle, 2002). To be sure, adding language would greatly augment mutual understanding. If conscious thought enables the person to simulate the mental states of others, this could be responsible for many vital aspects of human social life.

Culture depends on shared assumptions and shared knowledge. Groups have rules, norms, values, and morals, as well as shared goals. Members can interact without understanding each other's 
mental states, but appreciating the mental states of others (including the shared beliefs and assumptions) would enable vast improvements in the possibilities for collective action and interaction (e.g., de Waal, 2008). Proponents of seeing many animals as cultural creatures typically recognize that animal culture depends almost entirely on imitation, insofar as intentional teaching is essentially unknown among them (e.g., de Waal, 2001; Donald, 2002; Tomasello, 1999). Intentional teaching, however, may require much more in the way of understanding another's mental states, insofar as the teacher must understand the student's lack of understanding and devise ways of changing the student's mental condition. As evidence, people adjust their language to a level appropriate to those they are teaching - but occupying conscious thought undermines that process (Roßnagel, 2000). Hence, conscious simulation facilitates teaching.

Human social life benefits from much more varied and flexible prosocial behavior than is found in most other species. Indeed, some have argued that altruistic behavior toward nonrelatives is essentially unknown in other animals, including pretty much all nonhuman primates (Hammerstein, 2003; Stevens \& Hauser, 2004), whereas humans perform many acts of kindness and generosity toward nonkin, toward strangers, and, in some cases, toward people they will never meet.

What gets humans to help others? Empathy is mental (including emotional) simulation of the inner states of others, and it is a vital contributor to human prosocial behavior: People imagine the mental and emotional states of others and are moved by that empathic connection to be generous and helpful (Batson, Duncan, Ackerman, Buckley, \& Birch, 1981; Batson et al., 1988; Coke, Batson, \& McDavis, 1978; Krebs, 1975). Some have suggested that empathy begins almost upon birth, given the fact that 1-day old human babies cry when they hear other babies cry (Hoffman, 1981, 1982). Whether those responses are full-fledged empathy is debatable, but beyond any doubt, humans continue to develop empathic responses, and these mediate prosocial acts (Batson et al., 1981; Twenge, Baumeister, DeWall, Ciarocco, \& Bartels, 2007).

Indeed, empathy probably contributes more than the motivation to help others. It also improves the quality of help. People can help others much more effectively if they understand the needs of others. The same is true for other forms of prosocial behavior such as cooperation and forgiveness. Even intentional teaching (another distinctively human phenomenon) is greatly facilitated insofar as the teacher intuits the pupil's mental state and knowledge.

Economic transactions are an important aspect of culture and a powerful engine of economic progress (G. Clark, 2007; Horan, Bulte, \& Shogren, 2005; McNeill, 1982; Sowell, 2008). Economic transactions would be difficult if not impossible without theory of mind. Economists regard it as axiomatic that all economic transactions require some degree of trust, insofar as each person relies on the other to give what has been promised, without fraud or exploitation. (When trust is low, such as in large illegal drug deals, the transactions are especially prone to go badly, and hence, parties are often reluctant to make them.) Such economic trust obviously requires theory of mind, for each party to rely on the other's promises.

Crucially, trust increases the risk of betrayal and exploitation, and so, economic agents must understand the other's perspective sufficiently to know how far to trust. Thus, they must be able to simulate the other's perspective and motives to transact effectively. Similar considerations apply to negotiations.

Economic transactions make apparent the value of another aspect of mental simulation for social interaction, namely, the mutual construction of a shared but not-yet-present reality. Trade typically proceeds based on offers of exchange, but these need to be mutually understood. We have emphasized that conscious thought enables people to tell their thoughts to others and thereby construct a shared understanding, without which economic trade and many other human interactions would be unthinkable.

Division of labor can be achieved without theory of mind, as ant colonies show, but it must rely on fixed action patterns and therefore is limited. Division of labor in human groups is considerably more flexible than in animal groups. Even chimpanzees have never been found to collaborate, in the sense of working together in complementary roles toward shared goals while communicating (Tomasello et al., 2005). When circumstances change the contingencies for group performance, groups with theory of mind can quickly adjust their behavior in the expectation that others will make parallel adjustments because the members understand that all share the common goal. Every individual in a modern culture is a member of many different groups throughout life and at any given time. In many groups, each member plays a different role and may even take on different roles within the same group over time (e.g., via workplace promotion). The capacity for humans to be so flexible when working together is most likely due in part to theory of mind. The work shared by groups in human culture is not based on fixed action patterns as it is with ant colonies. Rather, the division of labor is based essentially on shared understandings: Each person fulfills his or her duties because he or she understands and trusts that the others will fulfill theirs in turn and that together these will produce a jointly desired outcome. Moreover, as we have noted, talk is almost invariably conscious, and nearly all human group activities are facilitated by talk (H. Clark, 1996).

Thus, the ability to simulate the perspectives, experiences, and inner processes of others, although imperfect, contributes in multiple essential ways to human social life. Culture would remain at the most rudimentary and primitive levels without it.

\section{Decision Making}

The evolving complexity of human social life caused decisions to become increasingly complex and, hence, increasingly difficult. Almost certainly, nature had to equip cultural animals with a relatively more advanced and sophisticated decision-making apparatus than humanity's evolutionary forebears had if they were to make a cultural system succeed. We propose that conscious thought is part of that. The capacity for simulation would open up vastly new and improved possibilities for enlightened decision making, as compared to deciding based on Skinnerian reinforcement.

The capacity for simulation enables the person to contemplate each option at a choice point by imagining what would happen if he or she performed that action. The simple Skinnerian animal can at best have positive or negative associations to the action itself, based on past experience; more cognitively complex animals might form simple expectancies about the immediate outcomes of an act. The conscious simulator can envision not only the action 
but also its extended consequences, including possible responses by others, subsequent actions, and all their consequences. The person can therefore choose the action that promises to bring the best results in the long run, as opposed to choosing whichever action seems most immediately appealing.

Decision making could well take full advantage of the simulation capability. It would be useful to draw on knowledge and memory to simulate possible courses of action. The simulations might well produce unexpected or unwanted results, which the central executive could then consider when making its behavioral choice. Equally important and revealing, of course, is the fact that people typically talk over important decisions before making them, with various confidants, and such talk depends on conscious thought with shared simulations of alternatives and potential consequences.

\section{Self-Control}

Self-regulation is an important adaptation for culture because it allows people to alter their behavior to bring it into line with the rules, laws, norms, and other standards that the culture has. Selfregulation and self-control are especially needed when the self must decide between competing motivations, such as between an immediate, pressing temptation and a long-term goal or moral ideal. In such cases, the stimulus in the immediate present would seemingly have an almost overpowering advantage because it can activate desires and approach tendencies repeatedly and strongly.

Conscious simulation can however reduce and possibly nullify that advantage. By simulating distal outcomes, it can activate the relevant motivations. To pursue the example of motivational conflict, it can stoke desires for the long-term goal or desires to live up to the moral ideal, thus making them salient enough to compete with the tempting stimulus. The worker walking home with the week's wages may feel drawn to the saloon, but simulating the possible future consequences (getting home late drunk and broke, spouse angry, bills unpaid, sleeping on couch) may strengthen the competing inclination to continue homeward.

Understanding this function of consciousness helps resolve one of the paradoxical findings from Mischel's (e.g., Mischel, 1974) research on delay of gratification. In his studies with what has become known as the marshmallow test, children chose between a small immediate reward and a larger, delayed reward. Getting the larger one thus required resisting the temptation to take the immediate reward. He found that seeing the actual reward (e.g., a marshmallow) impaired self-control, in the sense that it made participants more likely to take the immediate reward. However, seeing a picture of the reward had the opposite effect of strengthening self-control and improving the capacity to delay gratification. This was puzzling because, in both cases, the child was looking at a marshmallow. If we understand the role of consciousness as supporting self-control by simulating the future outcome, however, we can resolve the contradiction. The picture differed from the actual stimulus in that the picture (itself a simulation of an actual marshmallow) strengthened the ability of consciousness to simulate the future outcome and thereby to resist the temptation to take the immediate reward.

\section{Self as Agent}

The study of self has flourished in social psychology but not in cognitive and neuroscientific research. This may reflect the latter's emphasis on specific processes and subroutines, of which the self is not one, in contrast to the former's emphasis on interpersonal interactions and relationships, in which identified selves are indispensable. In our view, the self is a constructed unity of the psyche-indeed, constructed mainly to meet the demands of social life. A solitary, solipsistic creature would not find much need to develop a self, except in the most rudimentary sense of unity of body. The job of the self is to relate the animal body to the social group. Culture requires more in the way of selfhood and identity than simpler social systems might. Conscious thought facilitates acting as a self, which is to say a unity that is mindful of its place in society, including roles and moral reputation.

The crosstalk function of conscious thought is useful for enabling the self to function in human social life. Without conscious thought, a stimulus might activate some motivation or impulse and produce a response that would be deemed antisocial by others, which could therefore jeopardize the person's position within the social group. Even today, rule-breakers are excluded from groups and relationships through such means as divorce, termination of employment, and imprisonment. Many of the actions that lead to such exclusion are presumably done as impulsive, selfish, or simply thoughtless responses to circumstances (e.g., Gottfredson \& Hirschi, 1990). Failing to consider all moral and legal ramifications of the possible action before doing it thus increases the risk of exclusion. Likewise, other undesirable consequences stem from acting without thinking first. People often have long-term goals and values that may conflict with their short-term impulses. In such cases, to act on the first impulse could again be maladaptive.

In contrast, if the person consciously simulates the action before doing it, all the various brain sites can contribute their relevant knowledge. All the inner voices can be heard, as it were, and this would include legal and moral scruples that might generally be slower to be activated than appetitive responses (and hence might otherwise fail to prevent a problematic action). Thus, the action chosen after conscious simulation is more likely to express all the contents of the psyche than actions done on first impulse-and therefore can more plausibly be taken as expressing the self.

Conscious simulations are thus crucial in enabling actions to reflect the full self rather than some mere part of it. Automatic responses do not necessarily reflect the full self, and the person may sincerely disavow them (e.g., "I didn't mean to do that"). The reason for such disavowals is presumably that the behavior was enacted simply on the basis of one isolated mental site responding to the stimulus and prescribing how to behave. The person might not have chosen to act that way had other knowledge structures (e.g., knowledge about relevant laws, moral rules, or possible consequences) had the chance to contribute their objections. In contrast to such straightforward and automatic responding, a conscious simulation broadcasts the potential action to the entire mind, precisely for the purpose of collecting the input from those other sites. Hence, an action that has been consciously simulated has in a sense been approved by the entire mind, which makes it much better able to express the full self.

Indeed, concepts of responsibility and accountability invoke the full self. To act responsibly is not simply to be held accountable; 
rather, it implies that the person considers the implications and consequences in advance of the action, in expectation of being held accountable. We have argued that this is part of what conscious thought is designed to do.

\section{Learning}

Learning is vital to human success, and the informational richness of culture entails that there is much to learn throughout life. Although it is obvious that learning occurs in even very simple animals and thus does not require the human powers of conscious thought, we propose that conscious thought can facilitate learning, such as through sharing of information and simulated replays of past events.

A crucial aspect of sequential thought is that it can be shared with others. This is made possible by language, the ability to think about others' intentions, and the ability to share and coordinate one's intentions jointly with others (Tomasello et al., 2005). Evidence from comparative psychology confirms that humans are unique in the extent to which they share and accumulate information with others. Chimpanzees are capable of some forms of sequential thought as evidenced by research on insightful problem solving (Köhler, 1925). They are limited, however, in their capacity to share those insights with others. Social learning in chimpanzees involves acquisition of knowledge about the physical environment (Nagell, Olguin, \& Tomasello, 1993). A chimpanzee who watches another use a tool to acquire food learns only that the tool has certain features. The learner will exhibit knowledge of those features when using the tool later, but it will rarely use the tool for the same purpose or in the same manner. Learning in nonhuman animals is thus slow (requiring direct observation) and imprecise (in that only some features of the skill or behavior are transferred). Learning in humans, on the other hand, benefits from shared intentions and ways of doing things (Tomasello, 1999). Conscious thought and the capacity for mental simulation enable humans to communicate ideas, beliefs, rules, and behaviors at a much higher fidelity than is seen in other species (Boesch \& Tomasello, 1998).

Many animals learn effectively from their experiences. However, the Skinnerian animal either learns from an event as it happens or never learns (though if initial learning happens, it may gain by being replayed during sleep). Roberts (2002) reviewed studies showing that animals can learn effectively if reinforcement is immediate, but learning diminishes sharply with even brief delays. Thus, in a classic study by Grice (1948), rats learned quickly if the behavior was rewarded immediately, but even a 5-s delay meant that learning required hundreds of trials, and a 10-s delay produced failure to learn even after a thousand trials.

In contrast, humans can learn from long-gone events if they replay them. Much human rumination seems likely to reflect a search to extract a meaningful lesson (Antrobus, Singer, \& Greenberg, 1966; Silver, Boone, \& Stone, 1983; Tait \& Silver, 1989; see also Ciarocco, Vohs, \& Baumeister, 2008). Humans can replay events from yesterday or last year and continue to learn from them. Yet replaying them requires the conscious mind to simulate the event from memory so as to ponder and learn more from it.

Counterfactual replays are arguably even more important than simple reruns and even more valuable for learning (see Epstude \& Roese, 2008). People replay important events mentally, changing what they might have said or done and imagining how the result would have been different. Such a capability renders moot the concept of one-trial learning because a single behavioral trial could result in many dozens of simulated experiences, indeed, encompassing an assortment of behaviors that would explore the entire set of contingencies. Obviously, counterfactual replaying of recent events relies heavily on the capacity for simulation because the person is essentially going back through the experience not as it was lived but as it might have been different. Indeed, effective learning will depend on accurate counterfactual replay (e.g., correctly predicting how someone else would have reacted if one had done $\mathrm{X}$ instead of $\mathrm{Y}$ ), and such accuracy would constitute a form of wisdom presumably to be honed through considerable actual social experience.

Recent work on emotion has increasingly distinguished the conscious emotional state from automatic, sometimes nonconscious affective responses (e.g., Winkielman \& Berridge, 2004; Zajonc, 1980). Moreover, the function of full-blown conscious emotional states may be other than for the direct initiation of behavior. There is much more evidence that emotions influence cognitions than that emotions influence behavior (Schwarz \& Clore, 2007). Some ostensible evidence of emotion causing behavior is actually confounded, as shown by further studies with additional controls (for a review, see Baumeister et al., 2007). For example, Bushman, Baumeister, and Phillips (2001) found that anger did not directly cause aggression unless people anticipated that aggressing would make them feel better. Moreover, when emotion does directly influence behavior, the results can be maladaptive and self-defeating (e.g., Leith \& Baumeister, 1996), which is another reason to doubt that that is its primary evolved function.

Thus, in a sense, the literature on emotion has faced a parallel dilemma to that faced by theory of consciousness: There are accumulating reasons to question whether the conscious emotional state serves the function of direct control of behavior, and there is good reason to think the proper function should be sought elsewhere. Instead, evidence suggests that conscious emotion may serve to stimulate conscious reflection on past and future events, thus away from the here and now (for a review, see Baumeister et al., 2007). Emotion might provide the impetus to cause people to reconsider and replay past events, thereby improving the prospects of learning from them. Negative emotions in particular stimulate counterfactual thinking (Roese, 1997; see also Johnson-Laird \& Oatley, 2000). Regret and guilt promote ruminating about recent experiences and increase the likelihood that people will learn a specific lesson from them (Baumeister, Stillwell, \& Heatherton, 1995; Crawford, McConnell, Lewis, \& Sherman, 2002). Negative emotion stimulates detail-oriented thinking, which seems necessary to extract a specific lesson from a complex social event that has turned out poorly (Schwarz \& Clore, 2007). The usefulness of conscious emotion for learning is also suggested by patterns of occurrence. In particular, people feel more frequent and more intense emotions when performing novel behaviors (in which learning is still likely) than when performing habitual ones (where learning has presumably stopped; Wood, Quinn, \& Kashy, 2002).

Another link between conscious emotion and learning is the so-called emotional modulation of memory (McGaugh, 2000): Emotionally impactful events and information are remembered better than neutral ones. Thus, if incoming information evokes a conscious emotional response, it is remembered better than emo- 
tionally neutral information even a year later (Bradley, Greenwald, Petry, \& Lang, 1992; see also Dolcos, LaBar, \& Cabeza, 2005). Moreover, the benefits of emotion seem to be specific to the relevant lesson, insofar as emotion improves memory for relevant information rather than other, irrelevant information available at the same time (Christianson \& Loftus, 1987, 1991; Wessel \& Merckelbach, 1998).

Earlier, we noted that conscious thought may facilitate processing of information in story form because narrative structure involves extended meaningful sequences of information. In humans, much social learning comes in the form of these stories, including novels, television shows, movies, short stories, poems, comic books, myths, parables, and gossip. In contrast, nearly all studies of nonconscious learning use simple stimuli and associations rather than narratives, raising the possibility that the nonconscious mind is simply not capable of benefiting by acquiring information in narrative form (e.g., Greenwald \& Liu, 1985). Narratives may thus require conscious thought and simulation. Indeed, the capacity to learn through role playing games and make believe that develops in childhood (e.g., D. G. Singer \& Singer, 1990) has been described as a precursor to narrative thought (J. L. Singer \& Singer, 2006). Children learn early on how to immerse themselves in imagined experiences, and the ability to process and understand narratives hinges on this ability (Mar \& Oatley, 2008; Zwaan, 1999). Role-playing games and narratives are also very social in nature. Children's make-believe games require coordinating with others, and the sharing of tales, rumors, and gossip seems to be a natural component of social sharing and learning (see Dunbar, 1996; see also Baumeister et al., 2004). Throughout human cultural history, considerable learning has been achieved and transmitted in narrative form.

\section{Automatization}

Another route to learning involves automatization. It is well established that many responses become increasingly automatic over time. It was formerly assumed that increased skill and automaticity occur as the same neural pathways needed to execute an action become used over and over and thereby become increasingly efficient (e.g., James, 1890), but evidence has established that this view is wrong. Instead, it appears that one set of processes is used to learn the skill, whereas a different set of processes elsewhere in brain and mind executes it after it has become routine (e.g., Langer \& Imber, 1979; Lieberman et al., 2002).

It may be overly simple to suggest that conscious simulation learns the action and then, ever after, the automatic system executes it. Conscious thought can only simulate the action at the macro level, whereas automatic responses must execute it at the micro level. Most obviously, conscious simulation does not extend down to the neuronal firings that are necessary to carry out the action. Still, it does seem clear that conscious simulation is used initially when learning a skill in ways that it ceases to be used once the behavior is well learned (Kimble \& Perlmuter, 1970).

In a sense, macro simulation is the prototype for whatever online control of behavior by consciousness there is. Going to the kitchen to get a beer is preceded by a conscious simulation of the action, but conscious thought does not direct the leg and foot movements that actually carry out the trip. The simulation is a macro-level summary of the action, while the minutiae are supplied by the automatic system. Thus, the conscious simulation likely did not imagine each step, but the automatic system must execute each one patiently. The simulation allows other memory sites to supply additional information and correctives (e.g., that the nephew has probably drunk all the beer in the refrigerator, but there may be some in the rec room).

Gollwitzer and his colleagues (Brandstätter et al., 2001; Gollwitzer, 1999; Gollwitzer \& Brandstätter, 1997) provided important evidence of the transfer from conscious direction to automatic execution. Many studies have shown that forming an implementation intention facilitates behavior and removes the need for further, conscious efforts (e.g., Gollwitzer, 1993). That is, having a conscious thought in the specific "if ... then" form enables the automatic system to execute the response whenever the antecedent condition (the if part of "if ... then") is encountered. The "if ... then" statement can be considered a highly specific and effective simulation, which accounts for its consistent superiority over broad goal intentions.

As one typical demonstration of the efficacy of specific simulations, Gollwitzer and Brandstätter (1997) asked participants to furnish a report during the Christmas holidays, when people are typically busy with many things. Some had only the intention to write the report, whereas others formed a specific plan to do it at a particular time and place. The specific plan tripled the likelihood that participants would actually write the report. Thus, the highly specific simulation contributed powerfully toward enabling the automatic system to carry out the action.

\section{Explanations}

Early in this article, we acknowledged that some major critiques of conscious output noted that people's explanations are sometimes wrong (Gazzaniga, 2003; Nisbett \& Wilson, 1977). To our view, these errors show that explanations are simulations. The mind invents them to provide a narrative, causal account of internal or external events. The mistakes arise because the conscious mind does not have direct access to the truth. Instead, it seeks understanding by making simulations, and often, it articulates these to others.

One important function of conscious output is to explain the self to others. People furnish accounts of their actions and reasons. If effective, these accounts can bring important social rewards, including social approval, validation, and even cooperation and support. Ineffective accounts can bring the opposite results, which can cause problems for the individual, up to and including expulsion from the group.

As noted above, Nisbett and Wilson (1977) provided a seemingly devastating criticism of people's ability to furnish accurate introspective accounts of their reasons for many actions and judgments. They proposed that when people explain themselves, they often simply use standard explanations from a stockpile of culturally accepted explanations. Yet this is only a criticism if one assumes that the goal of explaining oneself is to furnish an introspectively accurate account.

Instead, we suggest the goal of explaining oneself is to secure and maintain social rewards, including approval, validation, and acceptance by the group (e.g., Henriques, 2003). The true inner reasons for the action are therefore much less important than the ability to reconcile the actions with the values and norms of the 
group. Ultimately, survival and reproduction-the basic criteria of biological success - depend on maintaining social acceptance. Acceptance, in turn, depends far more on being able to explain oneself in ways others will accept than on being able to report every subtle causal influence on one's actions.

The so-called stockpile therefore deserves further consideration. As described by Nisbett and Wilson (1977), explaining actions by borrowing from the stockpile of culturally accepted explanations seems a lame and lazy substitute for knowing one's true reasons. Yet the stockpile has the advantage that its explanations are socially and culturally accepted, and so, if people can furnish an account of their actions in those terms, they are likely to have their actions validated by the social group.

Recent work on moral judgment has converged on a similar conclusion. Haidt (2001) has shown that people often make and express moral judgments based on affective intuitions rather than by reasoning from basic principles. For example, after condemning a brother and sister for an inconsequential act of incestuous sex, participants were often unable to furnish a principled basis for their judgment and retreated to statements like "I don't know why, I just know it's wrong!" Initially, Haidt's work was taken as impugning moral reasoning and suggesting that such reasoning served little or no function. In subsequent writings, however, he was at pains to point out that moral reasoning still served a valuable purpose, namely, explaining one's actions and judgments (even after the fact) in terms others would accept and validate (see Haidt, 2007). Thus, one's moral actions may be based on automatic responses and affective intuitions, but conscious moral reasoning is needed to reconcile these actions with the principles that other people uphold so as to maintain one's position in the group as a morally respected actor.

The output function of conscious talk also addresses the problem identified by Gazzaniga (2003). He pointed out that the conscious mind constantly invents explanations for events in the world and that some of these are demonstrably false. However, if one verbalizes one's conclusions, then others can debate them and point out their flaws. This, after all, is one of the most important adaptive functions of culture (e.g., Baumeister, 2005): Knowledge and information can be shared and advanced collectively, so that culture will make collective progress. The social correction of individual errors thus greatly reduces the problematic flaws in the capacity of the conscious mind to understand events. Indeed, for a group to succeed as a culture, it is probably best to have precisely the situation Gazzaniga depicts: plenty of individuals simulating and generating ideas and explanations, then discussing them, and collectively ascertaining and embracing the most plausible ones.

\section{Conclusion}

The purpose of this article is to respond to accumulating evidence and theory that have depicted conscious thought as weak, ineffective, and possibly nothing more than a feckless, trivial by-product of other processes. We began by noting five major critiques that have questioned the utility and pragmatic capabilities of conscious thought. Specifically, conscious thought is sometimes mistaken about inner processes and reasons for acting, may be too slow to initiate behavior, succumbs to false explanations of external events, is not needed to produce many important behaviors, and can be mistaken about whether it exerts control.
Those critiques are based on the assumption that the functions of conscious thought are for enhancing sensory input from the physical environment (and awareness of inner processes) and for directly controlling behavior. We have rejected that assumption and suggested that the primary functions of conscious thought lie elsewhere. Specifically, a central and prominent function of conscious thought is to enable the animal to participate in the culture by sharing information and understanding one's role in relation to others. In terms of input, conscious thought is not needed for getting sensory input, hearing words, or even knowing what the words mean. Rather, it is for comprehending the meaning that lies in the sequences of words. Conscious thought thus processes information that the brain and mind already have. Its contribution to the control of behavior is far upstream, so that direct control and execution are the province of automatic, nonconscious processes. The human conscious experience of the here and now is constructively simulated, but the special value of conscious thought can be seen more readily in its simulation of events away from the here and now. These include simulating past experiences, future possibilities, and other people's perspectives.

One might ask why these processes need to be conscious. After all, many simulations are unconscious. Yet consciousness does appear to be necessary for several things that bring crucial advantages. First, consciousness enables communication across different parts of the mind and brain, thereby allowing widely distributed processes to contribute. Being able to act on the basis of many different internal stores of information, rather than on one at a time, is one large advantage. Second, consciousness enables people to talk to each other about their mental events. We have noted the irony that many theories use the ability to report on mental states as the operational definition of consciousness but that they seem to overlook the possibility that being able to report on mental states is precisely the point. By talking about one's thoughts, one can draw on the knowledge of the group, as well as make collective decisions and plan or reconsider group action. The selection advantage of being able to communicate with a human group is enormous, especially assuming that the group is a cultural one that operates by sharing information. Third, the mind seems unable to process various important forms of meaningfully sequential thought without consciousness. Consciousness therefore greatly improves the mind's power to comprehend and process information.

If our analysis is correct, then the five critiques, even if they were entirely valid, would still leave ample room for conscious thought to serve vital adaptive functions. The fact that selfexplanations sometimes resemble culturally shared assumptions rather than actual, idiosyncratic inner processes (Nisbett \& Wilson, 1977 ) is not surprising. Conscious thought is not for monitoring unconscious execution processes-but furnishing accounts that others will approve is one of its important jobs, and it creates explanations by simulating how the action most likely occurred rather than knowing from direct control. Its occasional mistakes about environmental events (e.g., Gazzaniga, 2000, 2003) again indicate merely that conscious thought is a construction, an educated guess, rather than a direct pipeline to the truth. Moreover, mistakes can be corrected socially, as long as people tell their inferences and explanations to others-and conscious thought is required for people to be able to talk and thereby to tell their thoughts to other people. Individuals make mistakes, but the group 
can converge on a correct and useful understanding, which is one immense benefit of information sharing in culture (Baumeister, 2005; Tomasello, 1999).

The observation that brain activity commences prior to conscious decisions to initiate motor movement (Libet, 1985) likewise poses no problem for the view we advocate. Direct control of motor movements is not the purpose of conscious thought. Meanwhile, of course, the brain has to work in advance to create a conscious thought. (Moreover, by instructing participants not to plan when to make their wrist or finger movement, the Libet procedures prohibited conscious thought from doing the one thing in that situation that it would be good for.)

Similarly, the program of research by Bargh and colleagues showing that many forms of behavior can be initiated without conscious decision is congenial to our assumption that the direct proximal execution of behavior is essentially always nonconscious. Nothing in that body of work precludes the possibility that simulating future behaviors, well before actually carrying them out, can be hugely helpful in enabling the behaviors to achieve their desired ends, especially in a highly complex social environment.

Last, the fact that the conscious mind can be mistaken about whether a response was caused by oneself or others (Wegner, 2002) can also be reconciled with our account. It affirms that any input from consciousness is upstream and that the conscious feeling of agency is itself a construction rather than a direct pipeline to reality. Still, it is probably right most of the time, even if it is a simulation.

We have emphasized that conscious thought is useful for simulating nonpresent realities. Still, our arguments also show how conscious thought can help with action in the (expanded) here and now even if, as its critics suggest, it is a bit too slow to operate the body in a fast-moving situation. Even in a crisis, there is potential value from engaging in mental construction and simulation that may be a few steps removed from direct sensory input and motor output. Attending to what is happening can broadcast the constructed input to the full brain and gain input from dispersed stores of knowledge. Thinking about what one is doing can enable one to simulate alternatives shortly before putting them into practice. Both of those are roughly what happens when one is hurrying somewhere, realizes one is going the wrong direction or has forgotten to bring the checkbook, and quickly designs a new ad hoc plan for action. In such cases, the gap between conscious process and direct online execution may be seconds rather than hours or days, but it is still real. We have emphasized simulating nonpresent realities because they make the offline functions of consciousness salient, but the application to the almost-present situation follows the same principles.

Human culture involves group action and group decision making. Animal social groups have limited capacity to make collective decisions wisely. Perhaps one animal initiates action and the others follow suit, as in a fleeing stampede or hunting charge. In contrast, the collective decision processes of human groups have contributed to human biological success and cultural progress. When facing a decision, human groups can mentally simulate the various courses of action and their likely consequences. Simulating each other's mental states enables them to discuss the options in relation to shared goals and values (and thereby to influence each other) and to correct errors in each other's thinking. They can discuss prior outcomes and relate counterfactual simulations of past events to the options they currently face. One person can influence others to avoid doing what may seem most immediately appealing, by warning about nonobvious potentially negative outcomes, and these simulated outcomes can override appealing cues from the immediate environment. All of this has worked much better for the human species than the group decision methods other animals use, which, after all, are still available to human beings if they would want them.

To return to the core question of what value is added by conscious thought, we have suggested the answer lies in fabrication and synthesis. The mind takes input from the senses, from social communication, from memory, and from its abstract knowledge, and then, it fashions moving sequences of thought. This process enables people (collectively) to use meaning to understand, predict, and control the world. It enables them to engage in logical, mathematical, and moral reasoning. It enables them to reflect on past and future, to appreciate realities in the context of counterfactuals and possibilities, and to use these thoughts to change their own inner mental programming by which their automatic system will execute behaviors. It enables them to understand each other and work together in vastly improved ways. Above all, it enables them to create and sustain their new kind of social system, namely, culture, which is where they learn to do all those other things. In these ways, conscious thought continues to make powerful contributions to the success of human culture and human life.

\section{References}

Ainslie, G. (2007). Foresight has to pay off the present moment. Behavioral and Brain Sciences, 30, 313-314.

Antrobus, J. S., Singer, J. L., \& Greenberg, S. (1966). Studies in the stream of consciousness: Experimental enhancement and suppression of spontaneous cognitive processes. Perceptual and Motor Skills, 23, 399-417.

Baars, B. J. (1988). A cognitive theory of consciousness. New York, NY: Cambridge University Press.

Baars, B. J. (1997). In the theater of consciousness: The workspace of the mind. New York, NY: Oxford University Press.

Baars, B. J. (2002). The conscious access hypothesis: Origins and recent evidence. Trends in Cognitive Sciences, 6, 47-52.

Baars, B. J. (2003, September 22). The global brainweb: An update on global workspace theory. Science \& Consciousness Review. Retrieved from http://sciconrev.org/2003/09/the-global-brainweb/

Baddeley, A. D., \& Hitch, G. J. (1974). Working memory. In G. A. Bower (Ed.), Recent advances in learning and motivation (Vol. 8, pp. 47-90). New York, NY: Academic Press.

Bargh, J. A. (1997). The automaticity of everyday life. In R. S. Wyer, Jr. (Ed.), Advances in social cognition: Vol. 10. The automaticity of everyday life (pp. 1-61). Mahwah, NJ: Erlbaum.

Bargh, J. A. (2006). What have we been priming all these years? On the development, mechanisms, and ecology of nonconscious social behavior. European Journal of Social Psychology, 36, 147-168.

Bargh, J. A. (2007). Social psychological approaches to consciousness. In P. D. Zelazo, M. Moscovitch, \& E. Thompson (Eds.), The Cambridge handbook of consciousness (pp. 555-569). New York, NY: Cambridge University Press.

Bargh, J. A., \& Chartrand, T. L. (1999). The unbearable automaticity of being. American Psychologist, 54, 462-479.

Bargh, J. A., Gollwitzer, P. M., Lee-Chai, A. Y., Barndollar, K., \& Trötschel, R. (2001). The automated will: Nonconscious activation and 
pursuit of behavioral goals. Journal of Personality and Social Psychology, 81, 1014-1027.

Barsalou, L. W. (2008). Grounded cognition. Annual Review of Psychology, 59, 617-645.

Barsalou, L. W., Niedenthal, P. M., Barbey, A. K., \& Ruppert, J. A. (2003). Social embodiment. In B. Ross (Ed.), The psychology of learning and motivation (Vol. 43, pp. 43-92). New York, NY: Academic Press.

Barthes, R. (1977). Image-music-text. New York, NY: Hill \& Wang.

Bartlett, F. C. (1932). Remembering: An experimental and social study. Cambridge, England: Cambridge University Press.

Batson, C. D., Duncan, B. D., Ackerman, P., Buckley, T., \& Birch, K. (1981). Is empathic emotion a source of altruistic motivation? Journal of Personality and Social Psychology, 40, 290-302.

Batson, C. D., Dyck, J. L., Brandt, J. R., Batson, J. G., Powell, A. L., McMaster, M. R., \& Griffitt, C. (1988). Five studies testing two new egoistic alternatives to the empathy-altruism hypothesis. Journal of Personality and Social Psychology, 55, 52-77.

Baumeister, R. F. (2005). The cultural animal: Human nature, meaning, and social life. New York, NY: Oxford University Press.

Baumeister, R. F., Masicampo, E. J., \& Vohs, K. D. (in press). Can consciousness cause behavior? Annual Review of Psychology.

Baumeister, R. F., Stillwell, A. M., \& Heatherton, T. F. (1995). Personal narratives about guilt: Role in action control and interpersonal relationships. Basic and Applied Social Psychology, 17, 173-198.

Baumeister, R. F., Vohs, K. D., DeWall, C. N., \& Zhang, L. (2007). How emotion shapes behavior: Feedback, anticipation, and reflection, rather than direct causation. Personality and Social Psychology Review, 11, 167-203.

Baumeister, R. F., Zhang, L., \& Vohs, K. D. (2004). Gossip as cultural learning. Review of General Psychology, 8, 111-121.

Bayne, T., \& Chalmers, D. (2003). What is the unity of consciousness? In A. Cleeremans (Ed.), The unity of consciousness: Binding, integration, and dissociation (pp. 23-58). Oxford, England: Oxford University Press.

Berger, P. L. (1967). The sacred canopy: Elements of a sociological theory of religion. Garden City, NY: Doubleday.

Block, N. (1995). On a confusion about a function of consciousness. Behavioral and Brain Sciences, 18, 227-272.

Block, N. (2007). Consciousness, accessibility and the mesh between psychology and neuroscience. Behavioral and Brain Sciences, 30, 481548 .

Boesch, C., \& Tomasello, M. (1998). Chimpanzee and human cultures. Current Anthropology, 39, 591-614.

Bogels, S. M., \& Zigterman, D. (2000). Dysfunctional cognitions in children with social phobia, separation anxiety, and generalized anxiety disorder. Journal of Abnormal Child Psychology, 28, 205-211.

Boyd, R., \& Richerson, P. J. (1985). Culture and the evolutionary process. Chicago, IL: University of Chicago Press.

Boyd, R., \& Richerson, P. J. (1995). Why does culture increase human adaptability? Ethology and Sociobiology, 16, 125-143.

Bradley, M. M., Greenwald, M. K., Petry, M. C., \& Lang, P. J. (1992). Remembering pictures: Pleasure and arousal in memory. Journal of Experimental Psychology: Learning, Memory, and Cognition, 18, 379390.

Brandstätter, V., Lengfelder, A., \& Gollwitzer, P. M. (2001). Implementation intentions and efficient action initiation. Journal of Personality and Social Psychology, 81, 946-960.

Bruner, J. S. (2002). Making stories: Law, literature, life. Cambridge, MA: Harvard University Press.

Buckner, R. L., \& Carroll, D. C. (2007). Self-projection and the brain. Trends in Cognitive Sciences, 11, 49-57.

Busby, J., \& Suddendorf, T. (2005). Recalling yesterday and predicting tomorrow. Cognitive Development, 20, 362-372.

Bushman, B. J., Baumeister, R. F., \& Phillips, C. M. (2001). Do people aggress to improve their mood? Catharsis beliefs, affect regulation opportunity, and aggressive responding. Journal of Personality and Social Psychology, 81, 17-32.

Byrne, R. W., \& Whiten, A. (1988). Machiavellian intelligence: Social expertise and the evolution of intellect in monkeys, apes and humans. Oxford, England: Clarendon Press.

Call, J., \& Tomasello, M. (2008). Does the chimpanzee have a theory of mind? 30 years later. Trends in Cognitive Sciences, 12, 187-192.

Carruthers, P. (2002). The cognitive functions of language. Behavioral and Brain Sciences, 25, 657-726.

Cartwright, R., \& Lamberg, L. (1992). Crisis dreaming: Using your dreams to solve your problems. New York, NY: HarperCollins.

Christianson, S., \& Loftus, E. F. (1987). Memory for traumatic events. Applied Cognitive Psychology, 1, 225-239.

Christianson, S., \& Loftus, E. F. (1991). Remembering emotional events: The fate of detailed information. Cognition \& Emotion, 5, 81-108.

Ciarocco, N. J., Vohs, K. D., \& Baumeister, R. F. (2008). Some good news about rumination: Task-focused thinking after failure facilitates performance improvement. Manuscript submitted for publication.

Clark, G. (2007). A farewell to alms: A brief economic history of the world. Princeton, NJ: Princeton University Press.

Clark, H. (1996). Using language. New York, NY: Cambridge University Press.

Cleeremans, A. X. (2006). Conscious and unconscious cognition: A graded, dynamic perspective. In Q. Jing, M. Rosenzweig, G. d'Ydewalle, H. Zhang, H.-C. Chen, \& K. Zhang (Eds.), Progress in psychological science around the world: Vol. 1. Neural, cognitive, and developmental issues (pp. 401-418). Hove, England: Psychology Press.

Coke, J. S., Batson, C. D., \& McDavis, K. (1978). Empathic mediation of helping: A two-stage model. Journal of Personality and Social Psychology, 36, 752-766.

Colgan, D. M. (1970). Effects of instructions on the skin resistance response. Journal of Experimental Psychology, 86, 108-112.

Cook, S. W., \& Harris, R. E. (1937). The verbal conditioning of the galvanic skin reflex. Journal of Experimental Psychology, 21, 202-210.

Corballis, M. C. (2003). From mouth to hand: Gesture, speech, and the evolution of right-handedness. Behavioral and Brain Sciences, 26, 199208.

Corballis, M. C. (2004). The origins of modernity: Was autonomous speech the critical factor? Psychological Review, 111, 543-552.

Corballis, M. C. (2009). The evolution of language. Annals of the New York Academy of Sciences, 1156, 19-43.

Costabile, K. A., \& Klein, S. B. (2008). Understanding and predicting social events: The effects of narrative construction on inference generation. Social Cognition, 26, 420-437.

Crawford, M. T., McConnell, A. R., Lewis, A. C., \& Sherman, S. J. (2002). Reactance, compliance, and anticipated regret. Journal of Experimental Social Psychology, 38, 56-63.

Crick, F. (1984). Function of the thalamic reticular complex: The searchlight hypothesis. Proceedings of the National Academy of Sciences, USA, 81, 4586-4590

Crick, F., \& Koch, C. (2003). A framework for consciousness. Nature Neuroscience, 6, 119-126.

Crowder, R. G., \& Wagner, R. K. (1991). The psychology of reading. New York, NY: Oxford University Press.

Damasio, A. R. (1999). The feeling of what happens: Body and emotion in the making of consciousness. New York, NY: Harcourt Brace.

Davidson, D. (1982). Rational animals. Dialectica, 36, 317-327.

Dehaene, S., Sergent, C., \& Changeux, J. (2003). A neuronal network model linking subjective reports and objective physiological data during conscious perception. Proceedings of the National Academy of Sciences, USA, 100, 8520-8525.

De Neys, W. (2006). Dual processing in reasoning: Two systems but one reasoner. Psychological Science, 17, 428-433.

Dennett, D. (1991). Consciousness explained. Boston, MA: Little, Brown. 
Deutsch, R., Gawronski, B., \& Strack, F. (2006). At the boundaries of automaticity: Negation as a reflective operation. Journal of Personality and Social Psychology, 91, 385-405.

de Waal, F. B. M. (2001). The ape and the sushi master. New York, NY: Basic Books.

de Waal, F. B. M. (2008). Putting the altruism back into altruism: The evolution of empathy. Annual Review of Psychology, 59, 279-300.

DeWall, C. N., Baumeister, R. F., \& Masicampo, E. J. (2008). Evidence that logical reasoning depends on conscious processing. Consciousness and Cognition, 17, 628-645.

Dijksterhuis, A., \& Nordgren, L. F. (2006). A theory of unconscious thought. Perspectives on Psychological Science, 1, 95-109.

Dolcos, F., LaBar, K. S., \& Cabeza, R. (2005). Remembering one year later: Role of the amygdale and the medial temporal lobe memory system in retrieving emotional memories. Proceedings of the National Academy of Sciences, USA, 102, 2626-2631.

Donald, M. (2002). A mind so rare: The evolution of human consciousness. New York, NY: Norton

Draine, S. C. (1997). Analytic limitations of unconscious language processing (Unpublished doctoral dissertation). University of Washington, Seattle, WA.

Dunbar, R. I. M. (1996). Deception as cause or consequence of language? Behavioral and Brain Sciences, 19, 548.

Dunbar, R. I. M. (1998). The social brain hypothesis. Evolutionary Anthropology, 6, 178-190.

Dunn, E. W., \& Laham, S. A. (2006). Affective forecasting: A user's guide to emotional time travel. In J. Forgas (Ed.), Hearts and minds: Affective influences on social cognition and behavior (pp. 177-193). New York, NY: Psychology Press.

Edelman, G. M. (2004). Wider than the sky: The phenomenal gift of consciousness. New Haven, CT: Yale University Press.

Eliade, M. (1978). A history of religious ideas: Vol. 1. From the stone age to the Eleusinian mysteries. Chicago, IL: University of Chicago Press.

Eliade, M. (1982). A history of religious ideas: Vol. 2. From Gautama Buddha to the triumph of Christianity. Chicago, IL: Chicago University Press.

Emerson, M. J., \& Miyake, A. (2003). The role of inner speech in task switching: A dual-task investigation. Journal of Memory and Language, $48,148-168$

Epstude, K., \& Roese, N. J. (2008). The functional theory of counterfactual thinking. Personality and Social Psychology Review, 12, 168-192.

Foulkes, D. (1985). Dreaming: A cognitive-psychological analysis. Hillsdale, NJ: Erlbaum.

Fox, M. C., Ericsson, K. A., \& Best, R. (2009). Do procedures for verbal reporting of thinking have to be reactive? A meta-analysis and recommendations for best reporting methods. Manuscript submitted for publication.

Frederick, S. (2005). Cognitive reflection and decision making. Journal of Economics Perspectives, 19, 25-42.

Galdi, S., Arcuri, L., \& Gawronski, B. (2008, August 22). Automatic mental associations predict future choices of undecided decision-makers. Science, 321, 1100-1102.

Gallese, V., \& Goldman, A. (1998). Mirror neurons and the simulation theory of mind-reading. Trends in Cognitive Sciences, 2, 493-501.

Garry, M., Manning, C. G., Loftus, E. F., \& Sherman, S. J. (1996). Imagination inflation: Imagining a childhood event inflates confidence that it occurred. Psychonomic Bulletin \& Review, 3, 208-214.

Gazzaniga, M. S. (2000). Cerebral specialization and interhemispheric communication: Does the corpus callosum enable the human condition? Brain, 123, 1293-1326.

Gazzaniga, M. S. (2003, January). The when, where, what, and why of conscious experience. Paper presented at the meeting of the National Institute on the Teaching of Psychology, St. Petersburg Beach, FL.
Gazzaniga, M. S. (2008). Human: The science behind what makes your brain unique. New York, NY: HarperCollins.

Gilbert, D. T., Pinel, E. C., Wilson, T. D., Blumberg, S. J., \& Wheatley, T. P. (1998). Immune neglect: A source of durability bias in affective forecasting. Journal of Personality and Social Psychology, 75, 617-638.

Glenberg, A. M. (1997). What memory is for. Behavioral and Brain Sciences, 20, 1-55.

Goldman, A. I. (1989). Interpretation psychologized. Mind and Language, 4, 161-185.

Gollwitzer, P. M. (1993). Goal achievement: The role of intentions. European Review of Social Psychology, 4, 141-185.

Gollwitzer, P. M. (1999). Implementation intentions: Strong effects of simple plans. American Psychologist, 54, 493-503.

Gollwitzer, P. M., \& Brandstätter, V. (1997). Implementation intentions and effective goal pursuit. Journal of Personality and Social Psychology, 73, 186-199.

Gordon, R. M. (1986). Folk psychology as simulation. Mind and Language, 1, 158-171.

Gottfredson, M. R., \& Hirschi, T. (1990). A general theory of crime. Stanford, CA: Stanford University Press.

Gould, S. J. (1977). Ever since Darwin: Reflections in natural history. New York, NY: Norton.

Graesser, A. C., Singer, M., \& Trabasso, T. (1994). Constructing inferences during narrative text comprehension. Psychological Review, 101, 371-395.

Greenwald, A. G., \& Liu, T. J. (1985). Limited unconscious processing of meaning. Bulletin of the Psychonomic Society, 23, 292-313.

Grice, G. R. (1948). The relation of secondary reinforcement to delayed reward in visual discrimination learning. Journal of Experimental Psychology, 38, 1-16.

Haggard, P., \& Cole, J. (2007). Intention, attention and the temporal experience of action. Consciousness and Cognition, 16, 211-220.

Haidt, J. (2001). The emotional dog and its rational tail: A social intuitionist approach to moral judgment. Psychological Review, 108, 814-834.

Haidt, J. (2007, May 18). The new synthesis in moral psychology. Science, 316, 998-1002.

Hall, C. S. (1955). The significance of the dream of being attacked. Journal of Personality, 24, 168-180.

Hall, C. S., \& Van De Castle, R. L. (1966). The content analysis of dreams. East Norwalk, CT: Appleton-Century-Crofts.

Hammerstein, P. (2003). Why is reciprocity so rare in social animals? A protestant appeal. In P. Hammerstein (Ed.), Genetic and cultural evolution of cooperation (pp. 83-93). Cambridge, MA: MIT Press.

Harris, P. L. (1991). The work of the imagination. In A. Whiten (Ed.), Natural theories of mind (pp. 283-304). Oxford, England: Blackwell.

Haselton, M. G., \& Buss, D. M. (2000). Error management theory: A new perspective on cross-sex mind reading. Journal of Personality and Social Psychology, 78, 81-91.

Hauser, M. D., Chomsky, N., \& Fitch, W. T. (2002, November 22). The faculty of language: What is it, who has it, and how did it evolve? Science, 298, 1569-1579.

Henriques, G. (2003). The tree of knowledge system and the theoretical unification of psychology. Review of General Psychology, 7, 150-182.

Herrmann, E., Call, J., Hernandez-Lloreda, M. V., Hare, B., \& Tomasello, M. (2007, September 7). Humans have evolved specialized skills of social cognition: The cultural intelligence hypothesis. Science, 317, $1360-1366$.

Hesslow, G. (2002). Conscious thought as simulation of behavior and perception. Trends in Cognitive Sciences, 6, 242-247.

Hoffman, M. L. (1981). Is altruism part of human nature? Journal of Personality and Social Psychology, 40, 121-137.

Hoffman, M. L. (1982). Development of prosocial motivation: Empathy and guilt. In N. Eisenberg (Ed.), The development of prosocial behavior (pp. 281-313). New York, NY: Academic Press. 
Horan, R. D., Bulte, E., \& Shogren, J. F. (2005). How trade saved humanity from biological exclusion: An economic theory of Neanderthal extinction. Journal of Economic Behavior and Organization, 58, 1-29.

Humphrey, N. (1986). The inner eye. London, England: Faber.

Humphrey, N. (2006). Seeing red: A study in consciousness. Cambridge, MA: Harvard University Press.

Humphrey, N. K. (1976). The social function of intellect. In P. P. G. Bateson \& R. A. Hinde (Eds.), Growing points in ethology (pp. 303317). Cambridge, England: Cambridge University Press.

Hupbach, A., Gomez, R., Hardt, O., \& Nadel, L. (2007). Reconsolidation of episodic memories: A subtle reminder triggers integration of new information. Learning \& Memory, 14, 47-53.

Izard, C. E. (2009). Emotion theory and research: Highlights, unanswered questions, and emerging issues. Annual Review of Psychology, 60, 1-25.

Jablonska, E., \& Lamb, M. J. (2005). Evolution in four dimensions: Genetic, epigenetic, behavioral, and symbolic variation in the history of life. Cambridge, MA: MIT Press.

James, W. (1890). The principles of psychology (Vols. 1-2). New York, NY: Holt.

Jeannerod, M. (2001). Neural simulation of action: A unifying mechanism for motor cognition. NeuroImage, 14, S103-S109.

Johnson-Laird, P. N., \& Oatley, K. (2000). The cognitive and social construction of emotions. In M. Lewis \& J. Haviland (Eds.), Handbook of emotions (pp. 458-475). New York, NY: Guilford Press.

Kane, M. J., Brown, L. H., McVay, J. C., Silvia, P. J., Myin-Germeys, I., \& Kwapil, T. R. (2007). For whom the mind wanders, and when: An experience-sampling study of working memory and executive control in daily life. Psychological Science, 18, 614-621.

Kimble, G. A., \& Perlmuter, L. C. (1970). The problem of volition. Psychological Review, 77, 361-384.

Klinger, E., \& Cox, W. M. (1987). Dimensions of thought flow in everyday life. Imagination, Cognition and Personality, 7, 105-128.

Köhler, W. (1925). The mentality of apes. Oxford, England: Harcourt, Brace.

Krebs, D. (1975). Empathy and altruism. Journal of Personality and Social Psychology, 32, 1134-1146.

Langer, E. J., \& Imber, L. G. (1979). When practice makes imperfect: Debilitating effects of overlearning. Journal of Personality and Social Psychology, 37, 2014-2024.

Lee, L., \& Ariely, D. (2006). Shopping goals, goal concreteness, and conditional promotions. Journal of Consumer Research, 33, 60-70.

Leith, K. P., \& Baumeister, R. F. (1996). Why do bad moods increase self-defeating behavior? Emotion, risk taking, and self-regulation. Journal of Personality and Social Psychology, 71, 1250-1267.

Libet, B. (1985). Unconscious cerebral initiative and the role of conscious will in voluntary action. Behavioral and Brain Sciences, 8, 529-566.

Libet, B. (2004). Mind time: The temporal factor in consciousness. Cambridge, MA: Harvard University Press.

Lieberman, M. D., Gaunt, R., Gilbert, D. T., \& Trope, Y. (2002). Reflexion and reflection: A social cognitive neuroscience approach to attributional inference. In M. P. Zanna (Ed.), Advances in experimental social psychology (pp. 199-249). San Diego, CA: Academic Press.

Mackay, D. G. (1973). Aspects of the theory of comprehension, memory and attention. Quarterly Journal of Experimental Psychology, 25, $22-40$.

Malle, B. F. (2002). The relation between language and theory of mind in development and evolution. In T. Givón \& B. F. Malle (Eds.), The evolution of language out of pre-language (pp. 265-284). Amsterdam, the Netherlands: John Benjamins.

Mar, R. A. (2004). The neuropsychology of narrative: Story comprehension, story production and their interrelation. Neuropsychologia, 42, $1414-1434$

Mar, R. A., \& Oatley, K. (2008). The function of fiction is the abstraction and simulation of social experience. Perspectives on Psychological Science, 3, 173-192.

Markovits, H., \& Nantel, G. (1989). The belief-bias effect in the production and evaluation of logical conclusions. Memory \& Cognition, 17, 11-17.

Mason, M. F., Norton, M. I., Van Horn, J. D., Wegner, D. M., Grafton, S. T., \& Macrae, C. N. (2007, January 19). Wandering minds: The default network and stimulus-independent thought. Science, 315, 393395.

McGaugh, J. L. (2000, January 14). Memory: A century of consolidation. Science, 287, 248-251.

McGuigan, F. J. (1970). Covert oral behavior during the silent performance of language tasks. Psychological Bulletin, 74, 309-326.

McGuigan, F. J., \& Bailey, S. C. (1969). Covert response patterns during the processing of language stimuli. Interamerican Journal of Psychology, 3, 289-299.

McGuigan, F. J., Keller, B., \& Stanton, E. (1964). Covert language responses during silent reading. Journal of Educational Psychology, 55, 339-343.

McNeill, W. H. (1982). The pursuit of power. Chicago, IL: University of Chicago Press.

Mead, G. H. (1934). Mind, self, \& society. Chicago, IL: University of Chicago Press.

Mele, A. R. (2009). Effective intentions: The power of conscious will. New York, NY: Oxford University Press.

Mendl, M., \& Paul, E. S. (2004). Consciousness, emotion, and animal welfare: Insights from cognitive science, Animal Welfare, 13, 17-25.

Miller, G. A. (1956). The magical number seven, plus or minus two: Some limits on our capacity for processing information. Psychological Review, 63, 81-97.

Mischel, W. (1974). Processes in delay of gratification. Advances in Experimental Social Psychology, 7, 249-292.

Mishkin, M., Ungerleider, L. G., \& Macko, K. A. (1983). Object vision and spatial vision: Two cortical pathways. Trends in Neuroscience, 6, 414417.

Miyake, A., Emerson, M. J., Padilla, F., \& Ahn, J. (2004). Inner speech as a retrieval aid for task goals: The effects of cue type and articulatory suppression in the random task cuing paradigm. Acta Psychologica, 115, $123-142$.

Morsella, E. (2005). The function of phenomenal states: Supramodular interaction theory. Psychological Review, 112, 1000-1021.

Nagell, K., Olguin, R. S., \& Tomasello, M. (1993). Processes of social learning in the tool use of chimpanzees (Pan troglodytes) and human children (Homo sapiens). Journal of Comparative Psychology, 107, $174-186$.

Niedenthal, P. M., Winkielman, P., Mondillon, L., \& Vermeulen, N. (2009). Embodiment of emotion concepts. Journal of Personality and Social Psychology, 96, 1120-1136.

Nisbett, R. E., \& Wilson, T. D. (1977). Telling more than we can know: Verbal reports on mental processes. Psychological Review, 84, 231-259.

Norem, J. K., \& Cantor, N. (1986). Defensive pessimism: Harnessing anxiety as motivation. Journal of Personality and Social Psychology, 51, $1208-1217$.

Ottaviani, R., \& Beck, A. T. (1987). Cognitive aspects of panic disorders. Journal of Anxiety Disorders, 1, 15-28.

Panksepp, J. (2005). Affective consciousness: Core emotional feelings in animals and humans. Consciousness and Cognition, 14, 30-80.

Pocket, S. (2004). Does consciousness cause behavior? Journal of Consciousness Studies, 11, 23-40.

Rescorla, R. A., \& Wagner, A. R. (1972). A theory of Pavlovian conditioning: Variations in the effectiveness of reinforcement and nonreinforcement. New York, NY: Appleton-Century-Crofts.

Revonsuo, A. (2000). The reinterpretation of dreams: An evolutionary hypothesis of the function of dreaming. Behavioral and Brain Sciences, $23,793-1121$ 
Roberts, W. A. (2002). Are animals stuck in time? Psychological Bulletin, 128, 473-489.

Roese, N. J. (1997). Counterfactual thinking. Psychological Bulletin, 121, 133-148.

Rosenbaum, D. A. (2002). Motor control. In H. Pashler (Series Ed.) \& S. Yantis (Vol. Ed.), Stevens' handbook of experimental psychology: Vol. 1. Sensation and perception (3rd ed., pp. 315). New York, NY: Wiley.

Roßnagel, C. (2000). Cognitive load and perspective-taking: Applying the automatic-controlled distinction to verbal communication. European Journal of Social Psychology, 30, 429-445.

Satpute, A. B., Fenker, D. B., Waldmann, M. R., Tabibnia, G., Holyoak, K. J., \& Lieberman, M. D. (2005). An fMRI study of causal judgments. European Journal of Neuroscience, 22, 1233-1238.

Sayette, M. A., Reichle, E. D., \& Schooler, J. W. (2009). Lost in the sauce: The effects of alcohol on mind wandering. Psychological Science, 20, $747-752$.

Sayette, M. A., Schooler, J. W., \& Reichle, E. D. (in press). Out for a smoke: The impact of cigarette craving on zoning-out during reading. Psychological Science.

Schacter, D. L., \& Addis, D. R. (2007, January 4). Constructive memory: The ghosts of past and future. Nature, 445, 27.

Schank, R. C., \& Abelson, R. P. (1995). Knowledge and memory: The real story. In R. S. Wyer (Ed.), Advances in social cognition (Vol. 8, pp. 1-95). Hillsdale, NJ: Erlbaum.

Schooler, J. W., Reichle, E. D., \& Halpern, D. V. (2004). Zoning out while reading: Evidence for dissociations between experience and metaconsciousness. In D. T. Levin (Ed.), Thinking and seeing: Visual metacognition in adults and children (pp. 203-226). Cambridge, MA: MIT Press.

Schwarz, N., \& Clore, G. L. (2007). Feelings and phenomenal experiences. In A. W. Kruglanski \& E. T. Higgins (Eds.), Social psychology: Handbook of basic principles (pp. 385-407). New York, NY: Guilford Press.

Seligman, M. E. P., \& Yellin, A. (1987). What is a dream? Behavior Research and Therapy, 25, 1-24.

Shanon, B. (2001). Against the spotlight model of consciousness. New Ideas in Psychology, 19, 77-84.

Shiffrin, R. M. (2008, July). The psychological basis of rationality: Examples from games. Paper presented at the Summer Institute on Bounded Rationality in Psychology and Economics, Berlin, Germany.

Shiffrin, R. M., \& Schneider, W. (1977). Controlled and automatic human information processing: II. Perceptual learning, automatic attending and a general theory. Psychological Review, 84, 127-190.

Silver, R. L., Boone, C., \& Stone, M. H. (1983). Searching for meaning in misfortune: Making sense of incest. Journal of Social Issues, 39, $21-$ 102.

Singer, D. G., \& Singer, J. L. (1990). The house of make-believe: Children's play and the developing imagination. Cambridge, MA: Harvard University Press.

Singer, J. L., \& Singer, D. G. (2006). Preschoolers' imaginative play as precursor of narrative consciousness. Imagination, Cognition, and Personality, 25, 97-117.

Smallwood, J., Franklin, M. S., Chin, J., Handy, T., \& Schooler, J. W. (2009). The future is in your hands: Evidence of a left hemisphere bias in naturally occurring prospective thought. Manuscript submitted for publication

Smallwood, J., Nind, L., \& O'Connor, R. C. (2009). When is your head at? An exploration of the factors associated with the temporal focus of the wandering mind. Consciousness and Cognition, 18, 118-125.

Smallwood, J., \& Schooler, J. W. (2006). The restless mind. Psychological Bulletin, 132, 946-958.

Smith, E. R., \& DeCoster, J. (1999). Associative and rule-based processing: A connectionist interpretation of dual-process models. In S. Chaiken \& Y. Trope (Eds.), Dual-process theories in social psychology (pp. 323-336). New York, NY: Guilford Press.
Smith, E. R., \& DeCoster, J. (2000). Dual-process models in social and cognitive psychology: Conceptual integration and links to underlying memory systems. Personality and Social Psychology Review, 4, 108 131.

Snyder, F. (1970). The phenomenology of dreaming. In L. Madow \& L. Snow (Eds.), The psychodynamic implications of the physiological studies on dreams (pp. 124-151). Springfield, IL: Charles C Thomas.

Sowell, T. (2008). Economic facts and fallacies. New York, NY: Basic Books.

Stevens, J. R., \& Hauser, M. D. (2004). Why be so nice? Psychological constraints on the evolution of cooperation. Trends in Cognitive Sciences, 8, 60-65.

Stickgold, R., Hobson, J. A., Fosse, R., \& Fosse, M. (2001, November 2). Sleep, learning, and dreams: Off-line memory reprocessing. Science, 294, 1052-1057.

Suddendorf, T. (2006, May 19). Behavior: Foresight and evolution of the human mind. Science, 312, 1006-1007.

Suddendorf, T., \& Busby, J. (2003). Mental time travel in animals? Trends in Cognitive Sciences, 7, 391-396.

Suddendorf, T., \& Busby, J. (2005). Making decisions with the future in mind: Developmental and comparative identification of mental time travel. Learning and Motivation, 36, 110-125.

Suddendorf, T., \& Corballis, M. C. (1997). Mental time travel and the evolution of the human mind. Genetic, Social, and General Psychology Monographs, 123, 133-167.

Suddendorf, T., \& Corballis, M. C. (2007). The evolution of foresight: What is mental time travel, and is it unique to humans? Behavioral and Brain Sciences, 30, 299-313

Tait, R., \& Silver, R. C. (1989). Coming to terms with major negative life events. In J. S. Uleman \& J. A. Bargh (Eds.), Unintended thought (pp. 351-382). New York, NY: Guilford Press.

Tomasello, M. (1999). The cultural origins of human cognition. Cambridge, MA: Harvard University Press.

Tomasello, M., Carpenter, M., Call, J., Behne, T., \& Moll, H. (2005). Understanding and sharing intentions: The origins of cultural cognition. Behavioral and Brain Sciences, 28, 675-735.

Tooby, J., \& DeVore, J. (1987). The reconstruction of hominid evolution through strategic modeling. In W. Kinzey (Ed.), The evolution of human behavior: Primate models (pp. 183-237). Albany, NY: SUNY Press.

Treisman, A. (1988). Features and objects: The fourteenth Bartlett Memorial Lecture. Quarterly Journal of Experimental Psychology: Human Experimental Psychology, 40(A), 201-237.

Treisman, A., \& Gelade, G. (1980). A feature integration theory of attention. Cognitive Psychology, 12, 97-136.

Treisman, A., \& Schmidt, H. (1982). Illusory conjunctions in the perception of objects. Cognitive Psychology, 14, 107-141.

Tulving, E. (2002). Episodic memory: From mind to brain. Annual Review of Psychology, 53, 1-25.

Tulving, E. (2005). Episodic memory and autonoesis: Uniquely human? In H. S. Terrace \& J. Metcalfe (Eds.), The missing link in cognition: Origins of self-reflective consciousness (pp. 3-56). New York, NY: Oxford University Press.

Twenge, J. M., Baumeister, R. F., DeWall, C. N., Ciarocco, N. J., \& Bartels, J. M. (2007). Social exclusion decreases prosocial behavior. Journal of Personality and Social Psychology, 92, 56-66.

Ungerleider, L. G., \& Mishkin, M. (1982). Two cortical visual systems. In D. Ingle, M. Goodale, \& R. Mansfield (Eds.), Analysis of visual behavior (pp. 549-586). Cambridge, MA: MIT Press

Vygotsky, L. S. (1962). Thought and language. Cambridge, MA: MIT Press.

Weatherford, J. (1997). The history of money. New York, NY: Three Rivers Press.

Wegner, D. M. (2002). The illusion of conscious will. Cambridge, MA: MIT Press. 
Wegner, D. M., Quillian, F., \& Houston, C. E. (1996). Memories out of order: Thought suppression and the disturbance of sequence memory. Journal of Personality and Social Psychology, 71, 680-691.

Wessel, I., \& Merckelbach, H. (1998). Memory for threat-relevant and threat-irrelevant cues in spider phobics. Cognition \& Emotion, 12, 93104

Winkielman, P., \& Berridge, K. C. (2004). Unconscious emotion. Current Directions in Psychological Science, 13, 120-123.

Winkielman, P., \& Schooler, J. (2008). Unconscious, conscious, and metaconscious in social cognition. In F. Strack \& J. Foerster (Eds.), Social cognition: The basis of human interaction (pp. 49-69). Philadelphia, PA: Psychology Press.

Wood, W., Quinn, J. M., \& Kashy, D. A. (2002). Habits in everyday life: Thought, emotion, and action. Journal of Personality and Social Psychology, 83, 1281-1297.
Wyer, R. S., Adaval, R., \& Colcombe, S. J. (2002). Narrative-based representations of social knowledge: Their construction and use in comprehension, memory and judgment. In M. P. Zanna (Ed.), Advances in experimental social psychology (Vol. 34, pp. 133-199). Boston, MA: Academic Press.

Yates, J. (1985). The content of awareness is a model of the world. Psychological Review, 92, 149-284.

Zajonc, R. B. (1980). Feeling and thinking: Preferences need no inferences American Psychologist, 35, 151-175.

Zwaan, R. A. (1999). Situation models: The mental leap into imagined worlds. Current Directions in Psychological Science, 8, 15-18.

Received September 3, 2008

Revision received February 3, 2010

Accepted February 9, 2010

\section{Members of Underrepresented Groups: Reviewers for Journal Manuscripts Wanted}

If you are interested in reviewing manuscripts for APA journals, the APA Publications and Communications Board would like to invite your participation. Manuscript reviewers are vital to the publications process. As a reviewer, you would gain valuable experience in publishing. The P\&C Board is particularly interested in encouraging members of underrepresented groups to participate more in this process.

If you are interested in reviewing manuscripts, please write APA Journals at Reviewers@apa.org. Please note the following important points:

- To be selected as a reviewer, you must have published articles in peer-reviewed journals. The experience of publishing provides a reviewer with the basis for preparing a thorough, objective review.

- To be selected, it is critical to be a regular reader of the five to six empirical journals that are most central to the area or journal for which you would like to review. Current knowledge of recently published research provides a reviewer with the knowledge base to evaluate a new submission within the context of existing research.

- To select the appropriate reviewers for each manuscript, the editor needs detailed information. Please include with your letter your vita. In the letter, please identify which APA journal(s) you are interested in, and describe your area of expertise. Be as specific as possible. For example, "social psychology" is not sufficient-you would need to specify "social cognition" or "attitude change" as well.

- Reviewing a manuscript takes time (1-4 hours per manuscript reviewed). If you are selected to review a manuscript, be prepared to invest the necessary time to evaluate the manuscript thoroughly. 